\title{
ENDS FOR MONOIDS AND SEMIGROUPS
}

\author{
DAVID A. JACKSON ${ }^{\bowtie}$ and VESNA KILIBARDA
}

(Received 22 May 2007; accepted 11 September 2008)

Communicated by M. G. Jackson

\begin{abstract}
We give a graph-theoretic definition for the number of ends of Cayley digraphs for finitely generated semigroups and monoids. For semigroups and monoids, left Cayley digraphs can be very different from right Cayley digraphs. In either case, the number of ends for the Cayley digraph does not depend upon which finite set of generators is used for the semigroup or monoid. For natural numbers $m$ and $n$, we exhibit finitely generated monoids for which the left Cayley digraphs have $m$ ends while the right Cayley digraphs have $n$ ends. For direct products and for many other semidirect products of a pair of finitely generated infinite monoids, the right Cayley digraph of the semidirect product has only one end. A finitely generated subsemigroup of a free semigroup has either one end or else has infinitely many ends.
\end{abstract}

2000 Mathematics subject classification: primary 20M99.

Keywords and phrases: ends, monoid and semigroup, Cayley digraphs.

\section{Ends for graphs and digraphs}

A digraph is a quadruple $\Gamma=\left(V_{\Gamma}, E_{\Gamma}, \iota_{\Gamma}, \tau_{\Gamma}\right)$ where $V=V_{\Gamma}$ is a set of vertices, $E=E_{\Gamma}$ is a set of edges and $\iota_{\Gamma}, \tau_{\Gamma}: E \rightarrow V$ are functions designating initial and terminal vertices for each edge. A graph is a quintuple $\Gamma=\left(V_{\Gamma}, E_{\Gamma}, \iota_{\Gamma}, \tau_{\Gamma}, \operatorname{inv}_{\Gamma}\right)$ where $\operatorname{inv}_{\Gamma}$ is a function $E \rightarrow E$ and we require axiomatically, for each $e \in E$, that $e \neq \operatorname{inv}_{\Gamma}(e)$, that $\operatorname{inv}_{\Gamma}\left(\operatorname{inv}_{\Gamma}(e)\right)=e$, that $\iota_{\Gamma}\left(\operatorname{inv}_{\Gamma}(e)\right)=\tau_{\Gamma}(e)$ and that $\tau_{\Gamma}\left(\operatorname{inv}_{\Gamma}(e)\right)=$ $\iota_{\Gamma}(e)$. We omit the subscripts on the functions $\iota_{\Gamma}$ and $\tau_{\Gamma}$ whenever context makes these unnecessary and we routinely write $e^{-1}$ for $_{\operatorname{inv}_{\Gamma}}(e)$.

When we imagine some geometric realization of a graph, we regard $e$ and $e^{-1}$ as occupying the same arc of points, but traversing these arcs in opposite directions. In a geometric realization for a digraph, each edge has an associated direction for traversal. We allow loops and multiple edges in graphs and digraphs.

A graph $\left(V_{\Upsilon}, E_{\Upsilon}, \iota_{\Upsilon}, \tau_{\Upsilon}\right.$, inv $\left._{\Upsilon}\right)$ is a subgraph of $\left(V_{\Gamma}, E_{\Gamma}, \iota_{\Gamma}, \tau_{\Gamma}\right.$, inv $\left._{\Gamma}\right)$ if $V_{\Upsilon}$ and $E_{\Upsilon}$ are subsets of $V_{\Gamma}$ and $E_{\Gamma}$, respectively, and the functions $\iota_{\Upsilon}, \tau_{\Upsilon}$ and $\operatorname{inv}_{\Upsilon}$ are the respective restrictions of the functions $\iota_{\Gamma}, \tau_{\Gamma}$ and $\operatorname{inv}_{\Gamma}$ to $E_{\Upsilon}$. Subdigraphs of

(C) 2009 Australian Mathematical Publishing Association Inc. 1446-7887/2009 \$16.00 
digraphs are defined analogously, but we will routinely use the word subgraph for both subgraphs and subdigraphs.

If $\Gamma$ is a digraph $(V, E, \iota, \tau)$ or a graph $\left(V, E, \iota, \tau,{ }^{-1}\right)$ and $V_{0}$ is a subset of $V$, then the full subgraph of $\Gamma$ on $V_{0}$ is the digraph $\Gamma_{0}=\left(V_{0}, E_{0}, \iota, \tau\right)$ or the graph $\Gamma_{0}=\left(V_{0}, E_{0}, \iota, \tau,{ }^{-1}\right)$ where $E_{0}$ consists of all of the edges in $E$ having both vertices in $V_{0}$. If $\mathfrak{F}$ is a subset of $V$, we write $\Gamma-\mathfrak{F}$ for the full subgraph of $\Gamma$ on $V-\mathfrak{F}$.

Suppose $\Gamma=(V, E, \iota, \tau)$ is a digraph and that $E^{-1}$ is a set in one-to-one correspondence with $E$, but disjoint from $E$. Then there is a graph $\overleftrightarrow{\Gamma}=\left(V, E \cup E^{-1}\right.$, $\left.\iota, \tau,{ }^{-1}\right)$ where we extend $\iota, \tau$ and ${ }^{-1}$ to $E \cup E^{-1}$ by $\iota\left(e^{-1}\right)=\tau(e), \tau\left(e^{-1}\right)=\iota(e)$ and $\left(e^{-1}\right)^{-1}=e$. If $\xi: \Gamma_{1} \rightarrow \Gamma_{2}$ is a morphism of digraphs, we obtain a graph morphism $\overleftrightarrow{\xi}: \overleftrightarrow{\Gamma}_{1} \rightarrow \overleftrightarrow{\Gamma}_{2}$ by setting $\overleftrightarrow{\xi}\left(e^{-1}\right)=(\xi(e))^{-1}$. It is then easily seen that $\leftrightarrow$ is a functor from the category of digraphs to the category of graphs.

Conversely, we obtain a digraph $(V, E, \iota, \tau)$ from a graph $\left(V, E, \iota, \tau,{ }^{-1}\right)$ by simply discarding the function ${ }^{-1}: E \rightarrow E$. This extends to a functor $U$ from the category of graphs to the category of digraphs. The functor $\leftrightarrow$ is a left adjoint to this functor $U$.

Suppose that $\Gamma=(V, E, \iota, \tau)$ is a digraph. A positive walk $\omega$ of length $n$ in $\Gamma$ is a sequence $\omega=\left(e_{1}, \ldots, e_{n}\right)$ of edges of $\Gamma$ with $\tau\left(e_{i}\right)=\iota\left(e_{i+1}\right)$ for $1 \leq i<n$. Similarly, if $\Gamma=\left(V, E, \iota, \tau,{ }^{-1}\right)$ is a graph, then a walk $\omega$ of length $n$ in $\Gamma$ is a sequence $\omega=\left(e_{1}, \ldots, e_{n}\right)$ of edges of $\Gamma$ with $\tau\left(e_{i}\right)=\iota\left(e_{i+1}\right)$ for $1 \leq i<n$. If $\xi: \Gamma_{1} \rightarrow \Gamma_{2}$ is a digraph morphism and $\omega=\left(e_{1}, \ldots, e_{n}\right)$ is a positive walk in $\Gamma_{1}$, then $\xi(\omega)=\left(\omega\left(e_{1}\right), \ldots, \omega\left(e_{n}\right)\right)$ is a positive walk in $\Gamma_{2}$. Likewise, if $\xi: \Gamma_{1} \rightarrow \Gamma_{2}$ is a graph morphism and $\omega$ is a walk in $\Gamma_{1}$, then the sequence $\xi(\omega)$ is a walk in $\Gamma_{2}$. It is more convenient to write just $e_{1} e_{2} \cdots e_{n}$ rather than $\left(e_{1}, e_{2}, \ldots, e_{n}\right)$ for a walk or positive walk $\omega$. We define a walk in the digraph $\Gamma=(V, E, \iota, \tau)$ to be a walk in $\overleftrightarrow{\Gamma}$ : that is, we allow edges to be traversed in either direction. The vertices of $\omega$ are the vertices $\iota\left(e_{i}\right)$ and $\tau\left(e_{i}\right)$ such that $e_{i}$ is an edge of $\omega$. The initial vertex of $\omega$ is $\iota\left(e_{1}\right)$, the initial vertex of $e_{1}$, and the terminal vertex of $\omega$ is $\tau\left(e_{n}\right)$, the terminal vertex of $e_{n}$. We allow at each vertex $v$ of a digraph $\Gamma$ or a graph $\Gamma$ an empty walk of length 0 having $v$ as both its initial and terminal vertex. A walk $\omega=e_{1} e_{2} \cdots e_{n}$ in a graph or in a digraph is a trail if whenever $e_{i}=e$ for some $i$, then $e_{j}$ is neither $e$ nor $e^{-1}$ for $j \neq i$. That is, identifying $e$ with $e^{-1}$, all of the edges on $\omega$ are distinct. A walk in a graph or in a digraph is a path if all of its vertices are distinct. An interior vertex on a path $\omega$ is any vertex on $\omega$ other than its initial and terminal vertices. We are largely concerned with paths rather than walks or trails. The reader should be aware that these words are not consistently defined in the literature and that the distinction between walks and paths is sometimes important in this work. In a digraph, a positive walk is a positive trail if all of its edges are distinct and is a positive path if all of its vertices are distinct.

A graph $\Gamma$ is connected if there is a path in $\Gamma$ from any vertex $v_{1}$ to any vertex $v_{2}$. We define a digraph $\Gamma$ to be connected if $\overleftrightarrow{\Gamma}$ is connected. A component of a graph or of a digraph $\Gamma$ is a maximal connected subgraph of $\Gamma$.

We write $|X|$ for the cardinality of a set $X$. If $v$ is a vertex in a graph or in a digraph, then indegree $(v)$, outdegree $(v)$ and degree $(v)$ are defined respectively 
by indegree $(v)=|\{e \mid \tau(e)=v\}|$, outdegree $(v)=|\{e \mid \iota(e)=v\}|$ and degree $(v)=$ indegree $(v)+\operatorname{outdegree}(v)$. A graph is locally finite if each vertex has finite degree. It is usual practice to define the number of ends of a graph only for locally finite graphs. For finitely generated semigroups and monoids we define the number of ends for Cayley graphs which may have vertices with infinite indegree.

We state several possible definitions for the number of ends of a graph and additional possible definitions for the number of ends of a digraph. For all of these definitions, $\Gamma$ is a graph or digraph, $\mathfrak{F}$ a finite set of vertices of $\Gamma$ and, for various subscripts $x, \mathfrak{C}_{x}=\mathfrak{C}_{x}(\Gamma-\mathfrak{F})$ is some set whose elements are infinite components, $C$, of $\Gamma-\mathfrak{F}$. For each subscript $x$, we define a number, $\mathrm{e}_{x}(\Gamma)$, of ends of $\Gamma$ by

$$
\mathrm{e}_{x}(\Gamma)=\max _{\mathfrak{F} \subseteq V, \mathfrak{F} \text { finite }}\left|\mathfrak{C}_{x}(\Gamma-\mathfrak{F})\right| .
$$

When $\mathfrak{C}_{x}$ and $\mathrm{e}_{x}$ are defined for graphs rather than for digraphs, we extend the definition for $\mathrm{e}_{x}$ to digraphs by $\mathrm{e}_{x}(\Gamma)=\mathrm{e}_{x}(\overleftrightarrow{\Gamma})$ for any digraph $\Gamma$.

If $v_{1}, v_{2}$ are vertices in the graph $\Gamma$, then the distance $d_{\Gamma}\left(v_{1}, v_{2}\right)$ between $v_{1}$ and $v_{2}$ in $\Gamma$ is the length of the shortest path in $\Gamma$ from $v_{1}$ to $v_{2}$. This distance is not defined if $v_{1}$ and $v_{2}$ are in different components of $\Gamma$. A path $\pi$ having initial vertex $v_{1}$ and terminal vertex $v_{2}$ is a geodesic in $\Gamma$ if the length of $\pi$ is $d_{\Gamma}\left(v_{1}, v_{2}\right)$. In a digraph $\Gamma$, a positive path $\pi$ having initial vertex $v_{1}$ and terminal vertex $v_{2}$ is a digeodesic in $\Gamma$ if $\pi$ is a positive path of minimal length in $\Gamma$ from $v_{1}$ to $v_{2}$. There is a digeodesic in $\Gamma$ from $v_{1}$ to $v_{2}$ if and only if there is a positive path in $\Gamma$ from $v_{1}$ to $v_{2}$.

Suppose that $\Phi$ is a subgraph of a graph $\Gamma$ or a digraph $\Gamma$. Then it is easy to see that a path in $\Phi$ which is a geodesic or digeodesic in $\Gamma$ is also a geodesic or digeodesic in $\Phi$. However, a geodesic or digeodesic in $\Phi$ need not be a geodesic or digeodesic in $\Gamma$.

A graph $\Gamma$ has unbounded paths (geodesics) if for every natural number $n$ there is a path (geodesic) of length $n$ in $\Gamma$. A digraph $\Gamma$ has unbounded positive paths (digeodesics) if for every natural number $n$ there is a positive path (digeodesic) of length $n$ in $\Gamma$. A vertex $v$ in a graph $\Gamma$ initiates unbounded paths (geodesics) if for every natural number $n$ there is a path (geodesic) of length $n$ in $\Gamma$ with initial vertex $v$. A vertex $v$ in a digraph $\Gamma$ initiates unbounded positive paths (digeodesics) if for every natural number $n$ there is a positive path (digeodesic) of length $n$ in $\Gamma$ with initial vertex $v$. A vertex $v$ in a digraph $\Gamma$ terminates unbounded positive paths (digeodesics) if for every natural number $n$ there is a positive path (digeodesic) of length $n$ in $\Gamma$ with terminal vertex $v$.

To implement the definitions displayed above for $\mathrm{e}_{x}(\Gamma)$, we need to define various sets $\mathfrak{C}_{x}(\Gamma-\mathfrak{F})$ where $\Gamma$ is a graph or digraph and $\mathfrak{F}$ is a finite set of vertices of $\Gamma$. For the sake of brevity and generality, we state these definitions for $\mathfrak{C}_{x}(\Gamma)$ in terms of $\Gamma$ rather than $\Gamma-\mathfrak{F}$. For a graph $\Gamma$, we define

$$
\begin{aligned}
\mathfrak{C}_{\infty}(\Gamma) & =\{C \mid C \text { is a component of } \Gamma \text { having infinitely many vertices }\}, \\
\mathfrak{C}_{p}(\Gamma) & =\{C \mid C \text { is a component of } \Gamma \text { having unbounded paths }\}
\end{aligned}
$$


$\mathfrak{C}_{g}(\Gamma)=\{C \mid C$ is a component of $\Gamma$ having unbounded geodesics $\}$,

$\mathfrak{C}_{*}(\Gamma)=\{C \mid C$ contains a vertex which initiates unbounded paths $\}$,

$\mathfrak{C}_{\dagger}(\Gamma)=\{C \mid C$ contains a vertex which initiates unbounded geodesics $\}$.

Similarly, for a digraph $\Gamma$, we define

$$
\begin{aligned}
\mathfrak{C}_{+p}(\Gamma) & =\{C \mid C \text { is a component of } \Gamma \text { having unbounded positive paths }\} \\
\mathfrak{C}_{\delta}(\Gamma) & =\{C \mid C \text { is a component of } \Gamma \text { having unbounded digeodesics }\} \\
\mathfrak{C}_{*}(\Gamma) & =\{C \mid C \text { contains a vertex which initiates unbounded positive paths }\} \\
\mathfrak{C}_{*}(\Gamma) & =\{C \mid C \text { contains a vertex which terminates unbounded positive paths }\} \\
\mathfrak{C}_{\vec{\delta}}(\Gamma) & =\{C \mid C \text { contains a vertex which initiates unbounded digeodesics }\} \\
\mathfrak{C}_{\overleftarrow{\delta}}(\Gamma) & =\{C \mid C \text { contains a vertex which terminates unbounded digeodesics }\}
\end{aligned}
$$

\section{LEMMA 1. Let $\Gamma$ be a connected graph.}

(1) If $\Gamma$ has unbounded paths, then every vertex in $\Gamma$ initiates unbounded paths.

(2) If $\Gamma$ has unbounded geodesics, then every vertex in $\Gamma$ initiates unbounded geodesics.

PROOF. Let $\hat{v}$ be an arbitrary vertex in $\Gamma$. Write $|\pi|$ for the length of a walk $\pi$.

(1) For each natural number $n$, we want to show the existence of a path $\pi_{n}$ in $\Gamma$ with $\iota\left(\pi_{n}\right)=\hat{v}$ and $\left|\pi_{n}\right|=n$. Let $\chi_{n}$ be a path in $\Gamma$ with $\left|\chi_{n}\right|=2 n$. Since $\Gamma$ is connected, the distance $d_{\Gamma}(u, v)$ is defined and finite for any two vertices $u, v \in \Gamma$. Choose $v_{n}$ to be a vertex $v$ on $\chi_{n}$ for which $d_{\Gamma}(\hat{v}, v)$ is minimized as $v$ ranges over the vertices on $\chi_{n}$. Let $\gamma_{n}$ be a path with length $d_{\Gamma}\left(\hat{v}, v_{n}\right)$ from $\hat{v}$ to $v_{n}$. Write $\chi_{n}$ as $\left(\alpha_{n}\right)^{-1} \beta_{n}$ where $\iota\left(\alpha_{n}\right)=\iota\left(\beta_{n}\right)=v_{n}$. Since $\left|\chi_{n}\right|=2 n$, either $\left|\alpha_{n}\right| \geq n$ or $\left|\beta_{n}\right| \geq n$. Assume, without loss of generality, that $\left|\alpha_{n}\right| \geq n$, so $\left|\gamma_{n} \alpha_{n}\right| \geq n$. Observe that $\gamma_{n} \alpha_{n}$ must be a path by the minimality of $d_{\Gamma}\left(\hat{v}, v_{n}\right)$. Let $\pi_{n}$ be the initial subpath of $\gamma_{n} \alpha_{n}$ having length $n$.

(2) For each natural number $n$, we want to show the existence of a geodesic $\pi_{n}$ in $\Gamma$ with $\iota\left(\pi_{n}\right)=\hat{v}$ and $\left|\pi_{n}\right|=n$. Let $\chi_{n}$ be a geodesic in $\Gamma$ with $\left|\chi_{n}\right|=2 n$. Since $\Gamma$ is connected, we can find geodesics $\alpha_{n}, \beta_{n}$ in $\Gamma$ with $\iota\left(\alpha_{n}\right)=\iota\left(\beta_{n}\right)=\hat{v}, \tau\left(\alpha_{n}\right)=\iota\left(\chi_{n}\right)$ and $\tau\left(\beta_{n}\right)=\tau\left(\chi_{n}\right)$. Then $\left(\alpha_{n}\right)^{-1} \beta_{n}$ is a walk in $\Gamma$ from $\iota\left(\chi_{n}\right)$ to $\tau\left(\chi_{n}\right)$, hence $\left|\left(\alpha_{n}\right)^{-1} \beta_{n}\right| \geq 2 n$. But then either $\left|\alpha_{n}\right| \geq n$ or $\left|\beta_{n}\right| \geq n$. Without loss of generality, assume $\left|\alpha_{n}\right| \geq n$ and let $\pi_{n}$ be the initial subpath of $\alpha_{n}$ having length $n$.

\section{COROLlaRY 2. Let $\Gamma$ be any graph.}

(1) $\mathfrak{C}_{p}(\Gamma)=\mathfrak{C}_{*}(\Gamma)$ and $\mathfrak{C}_{g}(\Gamma)=\mathfrak{C}_{\dagger}(\Gamma)$.

(2) $\mathrm{e}_{p}(\Gamma)=\mathrm{e}_{*}(\Gamma)$ and $\mathrm{e}_{g}(\Gamma)=\mathrm{e}_{\dagger}(\Gamma)$.

PROOF. (1) It is clear that $\mathfrak{C}_{*}(\Gamma) \subseteq \mathfrak{C}_{p}(\Gamma)$ and $\mathfrak{C}_{\dagger}(\Gamma) \subseteq \mathfrak{C}_{g}(\Gamma)$. The reverse inclusions follow by applying Lemma 1 to connected components of $\Gamma$.

(2) By the first part, $\mathfrak{C}_{p}(\Gamma-\mathfrak{F})=\mathfrak{C}_{*}(\Gamma-\mathfrak{F})$ and $\mathfrak{C}_{g}(\Gamma-\mathfrak{F})=\mathfrak{C}_{\dagger}(\Gamma-\mathfrak{F})$ for any finite subset $\mathfrak{F}$ of vertices of $\Gamma$. 


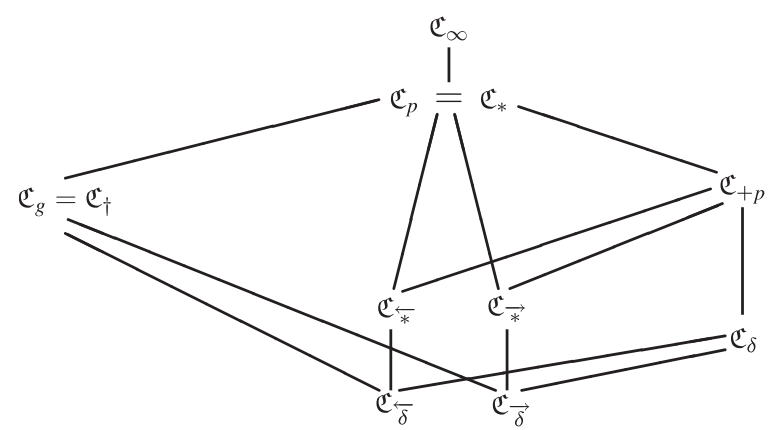

FIGURE 1. Some subset inclusions for $\mathfrak{C}_{\infty}$.

In the next six examples, the subscripts $\infty, p, g, *$ and $\dagger$ are graph subscripts while the subscripts $+p, \delta, \vec{*}, \overleftarrow{*}, \vec{\delta}$ and $\overleftarrow{\delta}$ are digraph subscripts. The subscripts $\vec{*}$ and $\vec{\delta}$ are initial subscripts while $\overleftarrow{*}$ and $\overleftarrow{\delta}$ are terminal subscripts. For Cayley digraphs of semigroups and monoids, we generally ignore the two terminal subscripts. The following examples show that, except for the equalities in the corollary above, the definitions in our list are all distinct. We do not claim to have included all possible definitions.

Let $\Gamma$ be a digraph and $\mathfrak{F}$ a finite set of vertices in $\Gamma$. Write just $\mathfrak{C}_{x}$ for $\mathfrak{C}_{x}(\Gamma-\mathfrak{F})$. Every $\mathfrak{C}_{x}$ is a subset of $\mathfrak{C}_{\infty}$ and we have illustrated some fairly obvious subset inclusions in Figure 1.

Since, for example,

$$
\mathfrak{C}_{\vec{\delta}}(\Gamma-\mathfrak{F}) \subseteq \mathfrak{C}_{*}(\Gamma-\mathfrak{F}) \subseteq \mathfrak{C}_{*}(\Gamma-\mathfrak{F}) \subseteq \mathfrak{C}_{\infty}(\Gamma-\mathfrak{F})
$$

we have $\mathrm{e}_{\vec{\delta}}(\Gamma) \leq \mathrm{e}_{*}(\Gamma) \leq \mathrm{e}_{*}(\Gamma) \leq \mathrm{e}_{\infty}(\Gamma)$, with similar inequalities following from other inclusions. An important consequence is that, for nonterminal subscripts $x$, all the numbers $\mathrm{e}_{x}(\Gamma)$ have the same value if we have $\mathfrak{C}_{\vec{\delta}}(\Gamma-\mathfrak{F})=\mathfrak{C}_{\infty}(\Gamma-\mathfrak{F})$ for every finite set $\mathfrak{F}$ of vertices in $\Gamma$. Similarly, for noninitial subscripts $x$, all the numbers $\mathrm{e}_{x}(\Gamma)$ have the same value if we have $\mathfrak{C}_{\overleftarrow{\delta}}(\Gamma-\mathfrak{F})=\mathfrak{C}_{\infty}(\Gamma-\mathfrak{F})$ for every finite set $\mathfrak{F}$ of vertices in $\Gamma$.

When a digraph $\Gamma$ has no multiple edges, it can be notationally very convenient to write $e=\left(v_{i}, v_{j}\right)$ for the edge $e$ which has $\iota(e)=v_{i}$ and $\tau(e)=v_{j}$. We follow this convention in the following six examples. If $\Gamma$ is a digraph without multiple edges, then the dual digraph $\Gamma^{\mathrm{op}}$ has the same vertices as $\Gamma$ and $\left(v_{j}, v_{i}\right)$ is an edge in $\Gamma^{\mathrm{op}}$ if and only if $\left(v_{i}, v_{j}\right)$ is an edge in $\Gamma$. We write $\mathbb{N}$ for the natural numbers, $\mathbb{Z}$ for the integers and $\mathbb{Z}_{n}$ for the integers modulo $n$.

EXAMPLE 1 . For $z=r, a, s$, let $\Gamma_{z}$ be the digraph $\left(V, E_{z}, \iota, \tau\right)$ where

$$
\begin{aligned}
V & =\left\{v_{i} \mid i \in \mathbb{Z}\right\}, \\
E_{r} & =\left\{\left(v_{i}, v_{i+1}\right) \mid i \in \mathbb{Z}\right\}, \\
E_{a} & =\left\{\left(v_{2 j}, v_{2 j+1}\right),\left(v_{2 j}, v_{2 j-1}\right) \mid j \in \mathbb{Z}\right\}, \\
E_{s} & =\left\{\left(v_{i}, v_{i+1}\right),\left(v_{2 j}, v_{2 j+1}\right),\left(v_{2 k}, v_{2 k-1}\right) \mid i \leq-1, j \geq 0, k \geq 1\right\} .
\end{aligned}
$$




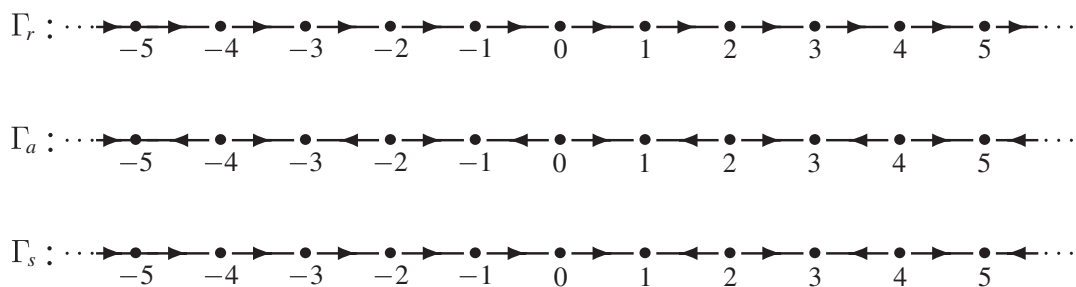

FIGURE 2. $\Gamma_{r}, \Gamma_{a}$ and $\Gamma_{s}$.

The subscripts $r, a, s$ are for right, alternating and split (see Figure 2). We observe first that $\overleftrightarrow{\Gamma}_{r}=\overleftrightarrow{\Gamma}_{a}=\overleftrightarrow{\Gamma}_{s}$, so that $\mathrm{e}_{x}\left(\Gamma_{r}\right)=\mathrm{e}_{x}\left(\Gamma_{a}\right)=\mathrm{e}_{x}\left(\Gamma_{s}\right)$ for any graph subscript $x$ and then that all of these have value 2. For $\Gamma_{r}$, we observe that $\mathrm{e}_{+p}\left(\Gamma_{r}\right)=\mathrm{e}_{\delta}\left(\Gamma_{r}\right)=2$, while $\mathrm{e}_{\overparen{*}}\left(\Gamma_{r}\right)=\mathrm{e}_{\vec{\delta}}\left(\Gamma_{r}\right)=\mathrm{e}_{\overleftarrow{*}}\left(\Gamma_{r}\right)=\mathrm{e}_{\overleftarrow{\delta}}\left(\Gamma_{r}\right)=1$. Since no positive path in $\Gamma_{a}$ has length greater than $1, \mathrm{e}_{x}\left(\Gamma_{a}\right)=0$ for every digraph subscript $x$. Similarly, $\mathrm{e}_{+p}\left(\Gamma_{s}\right)=\mathrm{e}_{\delta}\left(\Gamma_{s}\right)=\mathrm{e}_{\overleftarrow{*}}\left(\Gamma_{s}\right)=\mathrm{e}_{\overleftarrow{\delta}}\left(\Gamma_{s}\right)=1$, while $\mathrm{e}_{*}\left(\Gamma_{s}\right)=\mathrm{e}_{\vec{\delta}}\left(\Gamma_{s}\right)=0$

EXAMPLE 2. Let $\Gamma$ be the digraph with vertex set $V_{\Gamma}=\left\{v_{i, j} \mid i, j \in \mathbb{N}\right\}$ and edge set

$$
E_{\Gamma}=\left\{\left(v_{2 i, 1}, v_{2 i-1,1}\right),\left(v_{2 i, 1}, v_{2 i+1,1}\right),\left(v_{i, 2 j}, v_{i, 2 j-1}\right),\left(v_{i, 2 j}, v_{i, 2 j+1}\right) \mid i, j \in \mathbb{N}\right\} .
$$

Then $\mathrm{e}_{x}(\Gamma)=\infty$ for graph subscripts $x$ while $\mathrm{e}_{x}(\Gamma)=0$ for digraph subscripts $x$.

EXAMPLE 3. Let $n \geq 1$ be any natural number. For distinct symbols $h$ and $s_{i, j}$, let $V=\{h\} \cup\left\{s_{i, j} \mid 1 \leq i \leq n, j \geq 1\right\}$ be the set of vertices for a digraph $W_{n}$. Define the set $E$ of edges for $W_{n}$ by $E=\left\{\left(h, s_{i, 1}\right),\left(s_{i, j}, s_{i, j+1}\right),\left(s_{i, j}, h\right) \mid 1 \leq i \leq n, j \geq 1\right\}$. It is not difficult to see that $\mathrm{e}_{x}\left(W_{n}\right)=n$ except when $x$ is a terminal subscript, $\overleftarrow{*}$ or $\overleftarrow{\delta}$. For those two cases, $\mathrm{e}_{\leftarrow}\left(W_{n}\right)=1$ while $\mathrm{e}_{\overleftarrow{\delta}}\left(W_{n}\right)=0$. Similarly, $\mathrm{e}_{x}\left(W_{n}^{\mathrm{op}}\right)=n$ for noninitial subscripts $x$ while $\mathrm{e}_{*}\left(W_{n}^{\mathrm{op}}\right)=1$ and $\mathrm{e}_{\delta}\left(W_{n}^{\mathrm{op}}\right)=0$.

EXAMPLE 4. Let $n>1$ be a natural number. Let $V=\{c\} \cup\left\{v_{i, k} \mid i \in \mathbb{Z}_{n}, k \in \mathbb{N}\right\}$ be the set of vertices for a digraph $\Gamma_{n}$. Define the set $E$ of edges for $\Gamma_{n}$ by $E=\left\{\left(v_{i, k}, v_{i+1, k}\right),\left(v_{0, k}, c\right) \mid i \in \mathbb{Z}_{n}, k \in \mathbb{N}\right\}$. Since we can choose finite subsets $\mathfrak{F}$ of $V$ which exclude $c$, we see that $\mathrm{e}_{\infty}\left(\Gamma_{n}\right)=1$. For any other subscript $x, \mathrm{e}_{x}\left(\Gamma_{n}\right)=0$, since any trail in $\overleftrightarrow{\Gamma}_{n}$ with length greater than $2 n+2$ must pass through $c$ at least twice and thus cannot be a path.

EXAmple 5. Let $V=\{c\} \cup\left\{v_{j, k} \mid k \in \mathbb{N}, k \geq 3, j \in \mathbb{Z}_{k}\right\}$ be the set of vertices for a digraph $\Theta$. Define the set $E$ of edges for $\Theta$ by $E=\left\{\left(v_{j, k}, v_{j+1, k}\right),\left(v_{j, k}, c\right) \mid k \in \mathbb{N}\right.$, $\left.k \geq 3, j \in \mathbb{Z}_{k}\right\}$. Here $\mathrm{e}_{\infty}(\Theta)=\mathrm{e}_{p}(\Theta)=\mathrm{e}_{*}(\Theta)=\mathrm{e}_{+p}(\Theta)=\mathrm{e}_{\delta}(\Theta)=\mathrm{e}_{*}(\Theta)=1$. For the other five subscripts $x, \mathrm{e}_{x}(\Theta)=0$.

EXAmple 6. Let $X$ be any infinite set. Let the set of all finite subsets of $X$ be the set, $V=V_{\Gamma}$, of vertices for a digraph $\Gamma$. Define the set $E$ of edges for $\Gamma$ by $E=\{(A, B) \mid A, B \in V, B \subseteq A\}$. Then any positive path of length $n$ with initial 
vertex $A_{0} \in V$ corresponds to a chain of finite subsets $A_{n} \varsubsetneqq A_{n-1} \varsubsetneqq \cdots \varsubsetneqq A_{2} \varsubsetneqq A_{1}$ $\varsubsetneqq A_{0}$. Observe that there is a positive path in $\Gamma$ from $A$ to $B$ if and only if $B$ is a subset of $A$, that the longest positive path having $A$ as an initial vertex has length $|A|$ and that digeodesics in $\Gamma$ have length 1 .

Let $\mathfrak{F}=\left\{A_{\psi}\right\}_{\psi \in \Psi}$ be any finite subset of $V$ and define $A_{\mathfrak{F}}$ by $A_{\mathfrak{F}}=\bigcup_{\psi \in \Psi} A_{\psi}$. Then $A_{\mathfrak{F}}$ is also finite. Choose some element $x=x_{\mathfrak{F}} \in X-A_{\mathfrak{F}}$. Given any two vertices $B_{1}, B_{2} \in \Gamma-\mathfrak{F}$, let $B=B_{1} \cup B_{2} \cup\{x\}$. Then $B \in \Gamma-\mathfrak{F}$ and $\left(B, B_{1}\right),\left(B, B_{2}\right)$ are both edges in $\Gamma-\mathfrak{F}$, so $\Gamma-\mathfrak{F}$ is connected and geodesics in $\overleftrightarrow{\Gamma-\mathfrak{F}}$ have length at most 2

It is then clear that $\mathrm{e}_{\infty}(\Gamma)=\mathrm{e}_{p}(\Gamma)=\mathrm{e}_{*}(\Gamma)=\mathrm{e}_{+p}(\Gamma)=\mathrm{e}_{\leftarrow}(\Gamma)=1 \quad$ while $\mathrm{e}_{x}(\Gamma)=0$ for the other six subscripts $x$.

A set $X \subseteq M$ is a set of monoid generators for the monoid $M$ if every nonidentity element of $M$ can be written as a product of elements of $X$. A set $Y \subseteq M$ is a set of semigroup generators for the monoid $M$ if every element of $M$ can be written as a product of elements of $Y$.

For any semigroup $S$, we define $S^{1}$ to be the monoid $S \cup\{1\}$ where 1 is a new idempotent, not in $S$, and $1 \cdot s=s=s \cdot 1$ for every $s \in S$. For a semigroup homomorphism $f: S \rightarrow T$, we have a monoid homomorphism $f^{1}: S^{1} \rightarrow T^{1}$, extending $f$, if we define $f^{1}(1)=1$. Then ()$^{1}$ is a functor from the category of semigroups to the category of monoids and is the left adjoint to the forgetful functor from the category of monoids to the category of semigroups. If $\langle X: R\rangle$ is a semigroup presentation for $S$, then we can regard $\langle X: R\rangle$ as a monoid presentation for $S^{1}$, by allowing the empty word on $X$.

For a digraph $\Gamma$ and a semigroup $S$, let $\phi$ be a function from $S$ to $E=E_{\Gamma}$. Then the pair $(\Gamma, \phi)$ is a diagram over the semigroup $S, \phi$ is the label, or labelling function for the diagram and, for any edge $e \in E, \phi(e) \in S$ is the label of $e$. We extend the label by concatenation to a label on positive paths in $\Gamma$.

If $S$ is any semigroup with a finite set $X$ of generators and $s \in S$, we write $L_{X}(s)$ for the smallest positive integer $n$ such that $s=x_{i_{1}} x_{i_{2}} \cdots x_{i_{n}}$ with $x_{i_{j}} \in X$ for $1 \leq j \leq n$. Below, we will see that $L_{X}(s)$ is the length of a digeodesic from 1 to $s$ in either the right Cayley digraph $\Gamma_{r}\left(S^{1}, X\right)$ or the left Cayley digraph $\ell_{\ell}\left(X, S^{1}\right)$. When $M$ is a monoid and $X$ is a set of monoid generators for $M$, the identity 1 is the product of 0 elements from $X$ and we define $L_{X}(1)=0$. For any nontrivial $m \in M$, we write $L_{X}(m)$ for the smallest positive integer $n$ such that $m=x_{i_{1}} x_{i_{2}} \cdots x_{i_{n}}$ with $x_{i_{j}} \in X$ for $1 \leq j \leq n$. When $X$ is a set of monoid generators for the monoid $M$ and $m \in M$, we will see that $L_{X}(m)$ is the length of a digeodesic from 1 to $m$ in either the right Cayley digraph $\Gamma_{r}(M, X)$ or the left Cayley digraph ${ }_{\ell} \Gamma(X, M)$.

\section{Cayley digraphs for semigroups and monoids}

Suppose that $X \subseteq T$ is a set of semigroup generators for the semigroup $T$ or that $X \subseteq T$ is a set of monoid generators for the monoid $T$. The right Cayley digraph for 
$T$ with respect to $X$ is the digraph $\Gamma_{r}(T, X)=(V, E, \iota, \tau)$ where $V=T$,

$$
E=T \times X=\{(t, x) \mid t \in T, x \in X\}, \quad \iota((t, x))=t, \quad \tau((t, x))=t x .
$$

Dually, the left Cayley digraph for $T$ with respect to $X$ is the digraph ${ }_{\ell} \Gamma(X, T)=$ $(V, E, \iota, \tau)$ where $V=T$,

$$
E=X \times T=\{(x, t) \mid x \in X, t \in T\}, \quad \iota((x, t))=t, \quad \tau((x, t))=x t .
$$

The monoid Cayley digraphs are connected, but the semigroup Cayley digraphs need not be. For example, if $n$ is any natural number and $F_{n}$ is the free semigroup on the set $X_{n}=\left\{x_{1}, \ldots, x_{n}\right\}$ of generators, then both $\Gamma_{r}\left(F_{n}, X_{n}\right)$ and $\ell \Gamma\left(X_{n}, F_{n}\right)$ have $n$ components. For a given semigroup or monoid, these left and right Cayley digraphs can be quite dissimilar and will not in general have the same number of ends. Given a Cayley digraph $\Gamma$, the graph $\overleftrightarrow{\Gamma}$ is the Cayley graph. Any reference in this work to a positive path in a Cayley graph will always mean a positive path in the Cayley digraph. To define the corresponding right and left Cayley diagrams, we define a label $\phi$ by $\phi(t, x)=x$ or $\phi(x, t)=x$, respectively. We can regard this label as having values in the semigroup or monoid $T$ or as having values in the free semigroup or free monoid on the set $X$ of generators for $T$.

LEMMA 3. Suppose that $X$ is a finite set of monoid generators for the monoid T. Let $\Gamma$ be the right (left) Cayley digraph, $\Gamma_{r}(T, X)(\ell \Gamma(X, T))$. If $\mathfrak{F}$ is any finite set of vertices of $\Gamma$ and $C$ is an infinite component of $\Gamma-\mathfrak{F}$, then there is a vertex $\hat{v}$ in $C$ which initiates unbounded digeodesics.

Proof. The proof is the same for the right and left Cayley digraphs. We want to find a vertex $\hat{v}$ in $C$ and, for each natural number $n$, a digeodesic $\pi_{n} \in C$ with $\iota\left(\pi_{n}\right)=\hat{v}$ and $\left|\pi_{n}\right|=n$.

Since $C$ is infinite and there are only finitely many products of elements of $X$ having any fixed length, for each natural number $N$, we can find an element $v_{N}$ of $C$ such that $L_{X}\left(v_{N}\right) \geq N$. Select a digeodesic $\gamma_{N}$ in $\Gamma$ from 1 to $v_{N}$.

Suppose first that, for infinitely many values of $N$, the digeodesic $\gamma_{N}$ is contained in $C$. Then $1 \in C$. We let $\hat{v}=1$ and, for each natural number $n$, we select a digeodesic $\gamma_{N} \in C$ with $N \geq n$. Since $\left|\gamma_{N}\right|=L_{X}\left(v_{N}\right) \geq N$, the digeodesic $\gamma_{N}$ has length at least $n$ and we let $\pi_{n}$ be the initial subpath of $\gamma_{N}$ of length $n$.

Suppose then that $\gamma_{N}$ is in $C$ for only finitely many values of $N$. Then, for some sufficiently large $N_{0}$, the digeodesic $\gamma_{N}$ contains some vertex $a_{N} \in \mathfrak{F}$ whenever $N \geq N_{0}$. Let $\delta \mathfrak{F}=\{v \in \Gamma-\mathfrak{F} \mid$ there is an edge $e$ of $\Gamma$ with $\iota(e) \in \mathfrak{F}$ and $\tau(e)=v\}$. Since $\mathfrak{F}$ and $X$ are finite, $\delta \mathfrak{F}$ is finite. For $N \geq N_{0}$, we have $a_{N} \in \mathfrak{F}$ and $v_{N} \in C \subseteq$ $\Gamma-\mathfrak{F}$, so $\gamma_{N}$ contains at least one vertex which is in $\delta \mathfrak{F}$. For $N \geq N_{0}$, define $u_{N}$ to be the unique vertex $u$ on $\gamma_{N}$ such that $u \in \delta \mathfrak{F}$, but no vertex following $u$ on $\gamma_{N}$ is in $\delta \mathfrak{F}$. Since $\delta \mathfrak{F}$ is finite, there is at least one vertex $\hat{v}$ in $\delta \mathfrak{F}$ such that $\hat{v}=u_{N}$ for infinitely many different values of $N$. This is the vertex $\hat{v}$ required for the conclusion of the lemma. For any natural number $n$, choose $N$ so that $u_{N}=\hat{v}$ and $N \geq L_{X}(\hat{v})+n$. Then 
the terminal subpath of $\gamma_{N}$ from $\hat{v}$ to $v_{N}$ has length at least $n$. Let $\pi_{n}$ be the subpath of $\gamma_{N}$ having length $n$ and initial vertex $\hat{v}$. Since $\pi_{n}$ is a subpath of a digeodesic, $\pi_{n}$ is a digeodesic.

COROllary 4. Suppose that $X$ is a finite set of monoid generators for the monoid $T$. Let $\Gamma$ be the right Cayley digraph, $\Gamma_{r}(T, X)$. Then $\mathrm{e}_{x}(\Gamma)=\mathrm{e}_{\infty}(\Gamma)$ if $x$ is any of $p, g, *, \dagger,+p, \vec{*}$ or $\vec{\delta}$. Similarly, $\mathrm{e}_{x}(\Gamma)=\mathrm{e}_{\infty}(\Gamma)$ if $\Gamma$ is the left Cayley digraph $\ell \Gamma(X, T)$ and $x$ is any of these subscripts.

REMARK. The previous two results and arguments also hold for finitely generated semigroups.

EXAMPLE 7. If $X$ is a finite alphabet with $|X|>1, F$ is the free monoid generated by $X$ and $\Gamma$ is the corresponding right Cayley digraph, then $\mathrm{e}_{\infty}(\Gamma)=\infty$, but $\mathrm{e}_{*}(\Gamma)=$ $\mathrm{e} \overleftarrow{\delta}(\Gamma)=0$

\section{LEMMA 5.}

(a) Suppose that $M$ is a monoid with a finite set $X$ of monoid generators, that $\Gamma$ is the right (left) Cayley digraph for $M$ with respect to $X$ and that $\mathfrak{F}$ is any finite subset of $M$. Then $\Gamma-\mathfrak{F}$ has at most $1+|X||\mathfrak{F}|$ components.

(b) Suppose that $S$ is a semigroup with a finite set $X$ of generators, that $\Gamma$ is the right (left) Cayley digraph for $S$ with respect to $X$ and that $\mathfrak{F}$ is any finite subset of $S$. Then $\Gamma-\mathfrak{F}$ has at most $(1+|\mathfrak{F}|)|X|$ components.

PROOF. The proof is the same for the right and left Cayley digraphs.

(a) One of the components of $\Gamma-\mathfrak{F}$ might contain the identity element of $M$. We regard this potential component as accounting for the ' 1 ' in $1+|X||\mathfrak{F}|$. Suppose that $C$ is a component of $\Gamma-\mathfrak{F}$ which does not contain the identity element. Choose an element $m \in C$ for which $L_{X}(m)$ is minimal and, for the sake of notation, assume that $L_{X}(m)=k$. Choose a positive path of length $k$ from the identity element to $m$ in $\Gamma$ and let $v$ be the vertex occurring just before $m$ on this path. Observe that we must have $v \in \mathfrak{F}$ : otherwise $v \in \Gamma-\mathfrak{F}$ and the edge from $v$ to $m$ is in $\Gamma-\mathfrak{F}$, hence in $C$, but this contradicts our choice of $m \in C$ with $L_{X}(m)$ minimal. Then the edge from $v$ to $m$ is one of the $|X|$ edges having $v$ as its initial vertex and we can have at most $|X||\mathfrak{F}|$ such edges from vertices of $\mathfrak{F}$ to components of $\Gamma-\mathfrak{F}$ which do not contain the identity of $M$.

(b) Suppose that $S$ is a semigroup, that $\Gamma=\Gamma(S, X)$ and that $\mathfrak{F}$ is a finite subset of $S$. By part (a), $\Gamma\left(S^{1}, X\right)-\mathfrak{F}$ has at most $1+|X||\mathfrak{F}|$ components. One of these components contains the identity element of $S^{1}$. With this identity element removed in $\Gamma$, the remaining elements of this component partition into at most $|X|$ components of $\Gamma-\mathfrak{F}$. Hence $\Gamma-\mathfrak{F}$ has at most $|X|+|X||\mathfrak{F}|$ components.

It is not difficult to see that the bounds given in Lemma 5 are attained when we let $\mathfrak{F}$ be the set of all words of some fixed length on the free generators of a free monoid or a free semigroup. 
The following three corollaries of Lemma 5 are useful, expected and easy. We state and prove these for finitely generated monoids, but we remark that essentially the same proofs hold for finitely generated semigroups.

Corollary 6. Suppose that $X$ is a finite set of monoid generators for the monoid $T$. Let $\Gamma$ be the right (left) Cayley digraph, $\Gamma_{r}(T, X)(\ell \Gamma(X, T))$. If $T$ is infinite, then $\mathrm{e}_{\infty}(\Gamma) \geq 1$.

PROOF. For any finite $\mathfrak{F}, T$ is the disjoint union of $\mathfrak{F}$ and the components of $\Gamma-\mathfrak{F}$. By Lemma 5, there are only finitely many components, so at least one of these must be infinite.

COROLlary 7. Suppose that $X$ is a finite set of monoid generators for the monoid $T$. Let $\Gamma$ be the right (left) Cayley digraph, $\Gamma_{r}(T, X)\left({ }_{\ell} \Gamma(X, T)\right)$. If $\mathfrak{F}$ and $\hat{\mathfrak{F}}$ are finite subsets of $T$ with $\mathfrak{F} \subseteq \hat{\mathfrak{F}}$, then $\left|\mathfrak{C}_{\infty}(\Gamma-\mathfrak{F})\right| \leq\left|\mathfrak{C}_{\infty}(\Gamma-\hat{\mathfrak{F}})\right|$.

PROOF. Observe that each component of $\Gamma-\hat{\mathfrak{F}}$ must be contained in some component of $\Gamma-\mathfrak{F}$. Since $\Gamma-\hat{\mathfrak{F}}$ has only finitely many components, an infinite component, $C$, of $\Gamma-\mathfrak{F}$ can contain only finitely many components of $\Gamma-\hat{\mathfrak{F}}$ and finitely many elements of $\hat{\mathfrak{F}}$, so at least one of the components of $\Gamma-\hat{\mathfrak{F}}$ contained in $C$ must also be infinite.

EXAMPLE 8. The conclusion of Corollary 7 need not hold for arbitrary digraphs $\Gamma$. Let $\Gamma_{n}$ be the digraph of Example 4, $\mathfrak{F}$ any finite set of vertices of $\Gamma_{n}$ which does not include the vertex $c$ and $\hat{\mathfrak{F}}=\mathfrak{F} \cup\{c\}$. Then $\left|\mathfrak{C}_{\infty}\left(\Gamma_{n}-\mathfrak{F}\right)\right|=1$ but $\left|\mathfrak{C}_{\infty}\left(\Gamma_{n}-\hat{\mathfrak{F}}\right)\right|=0$.

COROLlary 8. Suppose that $X$ is a finite set of monoid generators for the monoid $T$. Let $\Gamma$ be the right (left) Cayley digraph, $\Gamma_{r}(T, X)(\ell \Gamma(X, T))$. For every natural number $n$, define $\mathfrak{F}_{n}$ to be $\left\{t \in T \mid L_{X}(t) \leq n\right\}$. Then $\mathfrak{F}_{n}$ is finite and $\mathrm{e}_{\infty}(\Gamma)=$ $\lim _{n \rightarrow \infty}\left|\mathfrak{C}_{\infty}\left(\Gamma-\mathfrak{F}_{n}\right)\right|$.

PROOF. It is clear that $\left|\mathfrak{F}_{n}\right| \leq \sum_{j=0}^{n}|X|^{j}$ and that

$$
\max _{\mathfrak{F}_{n}}\left|\mathfrak{C}_{\infty}\left(\Gamma-\mathfrak{F}_{n}\right)\right| \leq \mathrm{e}_{\infty}(\Gamma)=\max _{\mathfrak{F} \subseteq V_{\Gamma}, \mathfrak{F} \text { finite }}\left|\mathfrak{C}_{\infty}(\Gamma-\mathfrak{F})\right| .
$$

If $\mathrm{e}_{\infty}(\Gamma)$ is finite, then, for some finite $\mathfrak{F} \subseteq T,\left|\mathfrak{C}_{\infty}(\Gamma-\mathfrak{F})\right|=\mathrm{e}_{\infty}(\Gamma)$. Let $m=\max _{t \in \mathfrak{F}}\left\{L_{X}(t)\right\} . \quad$ Then $\mathfrak{F} \subseteq \mathfrak{F}_{n}$ for $n \geq m$, hence $\mathrm{e}_{\infty}(\Gamma)=\left|\mathfrak{C}_{\infty}(\Gamma-\mathfrak{F})\right| \leq$ $\left|\mathfrak{C}_{\infty}\left(\Gamma-\mathfrak{F}_{n}\right)\right|$ by Corollary 7 . It follows that $\lim _{n \rightarrow \infty}\left|\mathfrak{C}_{\infty}\left(\Gamma-\mathfrak{F}_{n}\right)\right|=\mathrm{e}_{\infty}(\Gamma)$.

If $\mathrm{e}_{\infty}(\Gamma)=\infty$, then for every natural number $k$ we can find a finite $\mathfrak{F} \subseteq T$ with $\left|\mathfrak{C}_{\infty}(\Gamma-\mathfrak{F})\right| \geq k$. Given such an $\mathfrak{F}$, let $n=\max _{t \in \mathfrak{F}}\left\{L_{X}(t)\right\}$. Then $\mathfrak{F} \subseteq \mathfrak{F}_{n}$ so $k \leq\left|\mathfrak{C}_{\infty}(\Gamma-\mathfrak{F})\right| \leq\left|\mathfrak{C}_{\infty}\left(\Gamma-\mathfrak{F}_{n}\right)\right|$ and $\lim _{n \rightarrow \infty}\left|\mathfrak{C}_{\infty}\left(\Gamma-\mathfrak{F}_{n}\right)\right|=\infty$.

LEMMA 9. If $X$ and $Y$ are finite sets of semigroup generators for the semigroup $S$, then $\mathrm{e}_{\infty}\left(\Gamma_{r}(S, X)\right)=\mathrm{e}_{\infty}\left(\Gamma_{r}(S, Y)\right)$ and $\mathrm{e}_{\infty}(\ell \Gamma(X, S))=\mathrm{e}_{\infty}(\ell \Gamma(Y, S))$.

If $X$ and $Y$ are finite sets of monoid generators for the monoid $M$, then $\mathrm{e}_{\infty}\left(\Gamma_{r}(M, X)\right)=\mathrm{e}_{\infty}\left(\Gamma_{r}(M, Y)\right)$ and $\mathrm{e}_{\infty}(\ell \Gamma(X, M))=\mathrm{e}_{\infty}(\ell \Gamma(Y, M))$. 
PROOF. We state the argument in terms of the right Cayley digraphs for semigroups: the argument for the left Cayley digraphs is dual and the monoid argument is essentially the same as the semigroup argument. Observe first that, by symmetry, it suffices to prove that $\mathrm{e}_{\infty}\left(\Gamma_{r}(S, X)\right)=\mathrm{e}_{\infty}\left(\Gamma_{r}(S, X \cup Y)\right)$, and second that we can reduce to the case of proving that $\mathrm{e}_{\infty}\left(\Gamma_{r}(S, X)\right)=\mathrm{e}_{\infty}\left(\Gamma_{r}(S, X \cup\{y\})\right)$ where $y \in Y$ by using induction on $|X \cup Y|-|X|$.

Suppose then that $S$ is a semigroup, that $X$ is a finite set of generators for $S$ and that $y \in S-X$. For brevity, write $\Gamma$ for the right Cayley digraph $\Gamma_{r}(S, X)$, and $\Gamma^{\prime}$ for the right Cayley digraph $\Gamma_{r}(S, X \cup\{y\})$. Then $S=V_{\Gamma}=V_{\Gamma^{\prime}}$ and $E_{\Gamma} \subset E_{\Gamma^{\prime}}$, so we may regard $\Gamma$ as a proper subgraph of $\Gamma^{\prime}$.

We want first to show that $\mathrm{e}_{\infty}\left(\Gamma^{\prime}\right) \leq \mathrm{e}_{\infty}(\Gamma)$. Let $\mathfrak{F}$ be any finite subset of $S$. It suffices to show that each of the finitely many infinite components of $\Gamma^{\prime}-\mathfrak{F}$ must contain one or more of the finitely many infinite components of $\Gamma-\mathfrak{F}$. Since all of the edges in $\Gamma-\mathfrak{F}$ are also edges in $\Gamma^{\prime}-\mathfrak{F}$, every component of $\Gamma-\mathfrak{F}$ is entirely contained in some one component of $\Gamma^{\prime}-\mathfrak{F}$. Since $\mathfrak{F}$ is finite and $\Gamma-\mathfrak{F}$ has only finitely many finite components, the union of the infinite components of $\Gamma-\mathfrak{F}$ contains all but finitely many of the vertices of $\Gamma$ and hence the union of the components of $\Gamma^{\prime}-\mathfrak{F}$ which contain infinite components of $\Gamma-\mathfrak{F}$ contains all but finitely many of the vertices in $V_{\Gamma^{\prime}}=V_{\Gamma}$. Hence, every infinite component of $\Gamma^{\prime}$ must be one of those which contains some infinite component of $\Gamma-\mathfrak{F}$.

Suppose next that $\mathrm{e}_{\infty}(\Gamma)$ is finite. Then there is a finite subset $\mathfrak{F}_{1}$ of $S$ such that $\Gamma-\mathfrak{F}_{1}$ has exactly $e_{\infty}(\Gamma)$ infinite components. Let $\mathfrak{F}_{+}$be the set of vertices in $\Gamma$ which can be reached from some vertex of $\mathfrak{F}_{1}$ by a positive path in $\Gamma$ having length at most $L_{X}(y)$. Observe that $\mathfrak{F}_{+}$is a finite set and let $\mathfrak{F}_{2}=\mathfrak{F}_{1} \cup \mathfrak{F}_{+}$. Then $\Gamma-\mathfrak{F}_{2}$ also has $\mathrm{e}_{\infty}(\Gamma)$ infinite components. Each infinite component, $C$, of $\Gamma-\mathfrak{F}_{2}$ is contained in some infinite component, $D$, of $\Gamma^{\prime}-\mathfrak{F}_{2}$. To show that $\Gamma^{\prime}-\mathfrak{F}_{2}$ has $\mathrm{e}_{\infty}(\Gamma)$ infinite components it suffices to show that no two distinct infinite components of $\Gamma-\mathfrak{F}_{2}$ are contained in the same infinite component of $\Gamma^{\prime}-\mathfrak{F}_{2}$. To this purpose, observe also that each infinite component, $C$, of $\Gamma-\mathfrak{F}_{2}$ is contained in an infinite component, $\hat{C}$, of $\Gamma-\mathfrak{F}_{1}$. Since $\mathfrak{F}_{1} \subseteq \mathfrak{F}_{2}$, it follows from Corollary 7 that $\left|\mathfrak{C}_{\infty}\left(\Gamma-\mathfrak{F}_{1}\right)\right|=$ $\left|\mathfrak{C}_{\infty}\left(\Gamma-\mathfrak{F}_{2}\right)\right|$ and hence that if $C_{1}, C_{2}$ are distinct infinite components of $\Gamma-\mathfrak{F}_{2}$, then $\hat{C}_{1}, \hat{C}_{2}$ are distinct infinite components of $\Gamma-\mathfrak{F}_{1}$. For the sake of obtaining a contradiction, suppose that two distinct infinite components, $C_{1}$ and $C_{2}$, of $\Gamma-\mathfrak{F}_{2}$ are contained in the same infinite component, $D$, of $\Gamma^{\prime}-\mathfrak{F}_{2}$. By choosing $C_{1}$ and $C_{2}$ to minimize the length of a path in $\Gamma^{\prime}-\mathfrak{F}_{2}$ connecting them, we may assume that there is an edge $f$ in $\Gamma^{\prime}-\mathfrak{F}_{2}$, labelled by $y$, having its initial vertex in $C_{1}$ and its terminal vertex in $C_{2}$. Write $v_{1}$ for the initial vertex of $f$ and $v_{2}$ for the terminal vertex of $f$. Then $v_{1}$ is also in $\hat{C}_{1}$ and $v_{2}$ is also in $\hat{C}_{2}$. Since the edge $f$ of $\Gamma^{\prime}$ has $v_{1}$ and $v_{2}$ for its endpoints, there is a path $\pi_{f}$ in $\Gamma$ having length $L_{X}(y)$ from $v_{1}$ to $v_{2}$. If $\pi_{f}$ connects $\hat{C}_{1}$ and $\hat{C}_{2}$ in $\Gamma-\mathfrak{F}_{1}$, then $\hat{C}_{1}=\hat{C}_{2}$ and $C_{1}=C_{2}$, so there must be some vertex on $\pi_{f}$ which occurs in $\mathfrak{F}_{1}$. But then $v_{2} \in \mathfrak{F}_{+} \subset \mathfrak{F}_{2}$, contradicting our assumption that $v_{2}$ is in a component of $\Gamma^{\prime}-\mathfrak{F}_{2}$. 
In the case where $\mathrm{e}(\Gamma)$ is infinite, we need to show that for every natural number $N$, there is a finite subset $\mathfrak{F}$ of $S$ such that $\Gamma^{\prime}-\mathfrak{F}$ has at least $N$ infinite components. Let $\mathfrak{F}_{1}$ be a finite subset of $S$ such that $\Gamma-\mathfrak{F}_{1}$ has at least $N$ distinct infinite components. Use $\mathfrak{F}_{1}$ and $L_{X}(y)$ to construct $\mathfrak{F}_{2}$ just as in the preceding paragraph. Then $\Gamma-\mathfrak{F}_{2}$ also has at least $N$ infinite components and, moreover, we can select $N$ of these which are contained in $N$ different components of $\Gamma-\mathfrak{F}_{1}$. It suffices to show that no two of these can be contained within the same component of $\Gamma^{\prime}-\mathfrak{F}_{2}$. For that, we may repeat the argument above using some edge $f$ and corresponding path $\pi_{f}$.

For a finitely generated semigroup $S$, we define $\mathcal{E}_{r}(S)$ and $\mathcal{E}_{\ell}(S)$ by $\mathcal{E}_{r}(S)=$ $\mathrm{e}_{\infty}\left(\Gamma_{r}(S, X)\right)$ and $\mathcal{E}_{\ell}(S)=\mathrm{e}_{\infty}(\ell \Gamma(S, X))$ for any finite set $X$ of semigroup generators for $S$. It is clear that we can state analogous definitions for $\mathcal{E}_{r}(M)$ and $\mathcal{E}_{\ell}(M)$ when $M$ is a finitely generated monoid.

We observe that when $M$ is a finitely generated monoid, the values for $\mathcal{E}_{r}(M)$ and $\mathcal{E}_{\ell}(M)$ do not change if we consider $M$ as a semigroup rather than as a monoid. By Lemma 9, we may assume that our set $X$ of monoid generators for $M$ includes the identity element of $M$. Then $X$ is also a set of semigroup generators for $M$. When we regard $M$ as a semigroup, we obtain the same right (left) Cayley digraph for $M$ with respect to $X$ that we obtain when we regard $M$ as a monoid.

If $G$ is a finitely generated group with a finite set $X$ of group generators, then $X \cup X^{-1}$ is a finite set of monoid generators for $G$. It is usual to consider a Cayley graph rather than a Cayley digraph for a group. Typically, this is the right Cayley graph, but it is isomorphic to the left Cayley graph. All vertices in the Cayley graph $\Gamma(G, X)$ have degree $2|X|$, so $\Gamma(G, X)$ is locally finite. There are numerous equivalent definitions for the number of ends of a finitely generated group (see [2, 3, 10-12]). One of these (see [10]) is to define the number of ends of $G$ to be $\lim _{n \rightarrow \infty}\left|\mathfrak{C}_{\infty}\left(\Gamma(G, X)-\mathfrak{F}_{n}\right)\right|$ where $\mathfrak{F}_{n}=\left\{g \in G \mid L_{X \cup X^{-1}}(g) \leq n\right\}$. By Corollary 8 , when a group $G$ is considered as a monoid, then its number of ends (considered as a group) is equal to both of the monoid values $\mathcal{E}_{r}(G)$ and $\mathcal{E}_{\ell}(G)$.

A function $\psi$ from a semigroup $S_{1}$ to a semigroup $S_{2}$ is an anti-homomorphism (see [1, Volume 1, p. 9]) if $\psi(a b)=\psi(b) \psi(a)$ for all $a, b \in S_{1}$. It is easy to see that the composition of two anti-homomorphisms is a homomorphism. An antihomomorphism is an anti-automorphism if $S_{1}=S_{2}$ and $\psi$ is a bijection.

For any semigroup $(S, \cdot)$ with associative multiplication $\cdot$ the dual semigroup $S^{\mathrm{op}}=$ $(S, *)$ has the same set of elements as $S$ and has associative multiplication $*$ defined by $s_{1} * s_{2}=s_{2} \cdot s_{1}$. Then the identity function on the set $S$ is an anti-automorphism between the semigroup $S$ and the semigroup $S^{\text {op }}$. Using this and composition, we may regard any anti-homomorphism from $S_{1}$ to $S_{2}$ as either a homomorphism from $S_{1}^{\text {op }}$ to $S_{2}$ or else as a homomorphism from $S_{1}$ to $S_{2}^{\text {op }}$. In particular, anti-automorphisms of $S$ correspond to isomorphisms between $S$ and $S^{\text {op }}$. Any set of generators for $S$ is also a set of generators for $S^{\text {op }}$ and whenever $X$ is a finite set of generators for $S$, then $\Gamma_{r}(S, X)={ }_{\ell} \Gamma\left(X, S^{\text {op }}\right)$, so $\mathcal{E}_{r}(S)=\mathcal{E}_{\ell}\left(S^{\text {op }}\right)$ and $\mathcal{E}_{\ell}(S)=\mathcal{E}_{r}\left(S^{\text {op }}\right)$. It is worth observing that ${ }^{\mathrm{op}}$ is a functor from the category of semigroups to itself. If $S_{1}$ and $S_{2}$ are 
semigroups and $f: S_{1} \rightarrow S_{2}$ is a homomorphism, then we may define $f^{\text {op }}: S_{1}^{\text {op }} \rightarrow S_{2}^{\text {op }}$ by $f^{\mathrm{op}}(s)=f(s)$ for $s \in S_{1}$. It is easy to verify that $f^{\text {op }}$ is a homomorphism. Generally, it is notationally convenient to suppress the distinction between $f$ and $f^{\text {op }}$.

Proposition 10. If the semigroup $S$ is isomorphic to $S^{\mathrm{op}}$, then $\mathcal{E}_{r}(S)=\mathcal{E}_{\ell}(S)$.

A semigroup $S$ is an inverse semigroup if for every element $s \in S$ there is a unique element, denoted $s^{-1}$, in $S$ such that $s s^{-1} s=s$ and $s^{-1} s s^{-1}=s^{-1}$. A monoid is an inverse monoid if it is an inverse semigroup when regarded as a semigroup. It is easy to see that $s s^{-1}$ and $s^{-1} s$ are idempotent elements in any inverse semigroup, and it is very well known (see [1] or [8]) that idempotents in an inverse semigroup commute with each other. From these, it follows easily that for $s, t \in S,(s t)^{-1}=t^{-1} s^{-1}$ and hence that ()$^{-1}$ is an anti-automorphism.

COROLLARY 11. If $T$ is a finitely generated inverse semigroup or a finitely generated inverse monoid, then $\mathcal{E}_{r}(T)=\mathcal{E}_{\ell}(T)$.

PROOF. As noted, the inversion operation is an anti-automorphism.

\section{Some constructions and examples}

We give three equivalent descriptions for the construction of a useful monoid $\hat{M}$ followed by some comments about the corresponding semigroup construction.

Suppose that $M$ and $T$ are monoids and that $M=S^{1}$ for some semigroup $S$, or equivalently that the identity element of $M$ is the only element of $M$ that is a left unit or a right unit. Define a multiplication $*$ on the set $T \times M$ by

$$
\left(t_{1}, m_{1}\right) *\left(t_{2}, m_{2}\right)= \begin{cases}\left(t_{1} t_{2}, m_{2}\right) & \text { if } m_{1}=1, \\ \left(t_{1}, m_{1} m_{2}\right) & \text { otherwise }\end{cases}
$$

We need the assumption that $M=S^{1}$ to prove that $*$ is associative. It is easy to see that $(1,1)$ is an identity element, so $(T \times M, *)$ is a monoid. We use $\hat{M}$ as a succinct notation for this monoid. If $T$ is also a monoid in which the identity element is the only left or right unit, then this assumption also holds for $\hat{M}$.

For the sake of notation, let $\left\langle A: R_{1}\right\rangle$ be a monoid presentation for $T$ and $\left\langle X: R_{2}\right\rangle$ a monoid presentation for $M$. Then the monoid $\hat{M}$ has monoid presentation

$$
\left\langle A \cup X \mid R_{1} \cup R_{2} \cup\{(x a, x) \mid a \in A, x \in X\}\right\rangle .
$$

We want to identify the ordered pair $(t, m)$ with the word $t m$ where $t$ is a word on $A$ and $m$ is a word on $X$. It is easy to show that every element of the presentation can be written in the form $t m$. It is more tedious to show that $t m=t^{\prime} m^{\prime}$ in $\hat{M}$ implies that $t=t^{\prime}$ in $T$ and that $m=m^{\prime}$ in $M$ : the assumption that $M=S^{1}$ for some semigroup $S$ is necessary for this uniqueness result.

Later in this paper we provide a more elaborate discussion of semidirect products of monoids. Define $\theta_{T} \in \operatorname{End}(T)$ to be the monoid endomorphism of $T$ which 
takes every element of $T$ to the identity element $1_{T}$ and write $\iota_{T}$ for the identity automorphism of $T$. Define a monoid homomorphism $\Phi_{0}: M \rightarrow \operatorname{End}(T)$ to be the monoid homomorphism which takes $1_{M}$ to $\iota_{T}$ and takes every other element of $M$ to $\theta_{T}$. Here, we need the assumption that $M=S^{1}$ to ensure that $\Phi_{0}$ is a homomorphism. Then the multiplication $*$ above and the monoid presentation above are the multiplication and a presentation for the monoid semidirect product $T \rtimes_{\Phi_{0}} M$. This approach to describing $\hat{M}$ has the notational advantage that we can conveniently describe different $\hat{M}$ s for different choices of $M$ and $T$. We utilize this notation in some examples.

If $S$ and $T$ are semigroups with semigroup presentations $\left\langle X: R_{2}\right\rangle$ and $\left\langle A: R_{1}\right\rangle$, respectively, then we can define a semigroup $\hat{S}$ having semigroup presentation

$$
\left\langle A \cup X \mid R_{1} \cup R_{2} \cup\{(x a, x) \mid a \in A, x \in X\}\right\rangle
$$

which is clearly analogous to the monoid presentation for $\hat{M}$ given above. We could also describe this semigroup $\hat{S}$ by defining the obvious multiplication on the set $(T \times S) \cup T \cup S$ or by recognizing $\hat{S}$ as the subsemigroup of nonidentity elements of the monoid semidirect product $T^{1} \rtimes_{\Phi_{0}} S^{1}$. We will not need the semigroup version of the following lemma.

LAYER LEMMA. Let $T$ be a finite monoid and $M$ a finitely generated monoid. Assume that $M=S^{1}$ for some semigroup $S$. Then $\mathcal{E}_{r}\left(T \rtimes_{\Phi_{0}} M\right)=|T| \mathcal{E}_{r}(M)$ and $\mathcal{E}_{\ell}\left(T \rtimes_{\Phi_{0}} M\right)=\mathcal{E}_{\ell}(M)$.

Proof. Let $X$ be a finite set of monoid generators for $M$ and let $A$ be a finite set of monoid generators for $T$. We write $x$ or $x_{j}$ for elements of $X$ and $a$ or $a_{k}$ for elements of $A$. Write $T=\left\{1_{T}=t_{1}, t_{2}, \ldots, t_{n}\right\}$ for some fixed ordering of the $|T|=n$ elements of $T$. We use $m, m_{1}, m_{2}$ as notation for arbitrary elements of $M$ and use $t, t_{i}, t_{i_{1}}, t_{i_{2}}$ as notation for arbitrary elements of $T$. We use $\hat{M}$ as an abbreviation for $T \rtimes_{\Phi_{0}} M$. We write just $t m, t$ and $m$ for elements $(t, m),\left(t, 1_{M}\right)$ and $\left(1_{T}, m\right)$ of $\hat{M}$ and we similarly identify $X$ with $\left\{1_{T}\right\} \times X$ and $A$ with $A \times\left\{1_{M}\right\}$.

Write $\Gamma$ for the right Cayley digraph $\Gamma_{r}(M, X)$ and $\hat{\Gamma}$ for the right Cayley digraph $\Gamma_{r}(\hat{M}, A \cup X)$. Here, it will be useful to regard $\Gamma$ and $\hat{\Gamma}$ as diagrams with labelled edges. For $1 \leq i \leq n$, we define the $i$ th layer in $\hat{\Gamma}$ to be the subdiagram $\Gamma_{i}$ of $\hat{\Gamma}$ having vertices $V_{i}=\left\{t_{i} m \mid m \in M\right\}$ and edges $E_{i}=\left\{f \mid f \in E_{\hat{\Gamma}}, \iota(f)=t_{i} m, \tau(f)=\right.$ $\left.t_{i} m x, x \in X\right\}$. Then, for each $i$ with $1 \leq i \leq n$, there is a diagram isomorphism $\phi_{i}: \Gamma \rightarrow \Gamma_{i}$, defined on vertices and edges by $\phi_{i}(m)=t_{i} m$, and $\phi_{i}(f)$ is the edge from $t_{i} m$ to $t_{i} m x$ with label $x$ if $f$ is the edge from $m$ to $m x$ having label $x$. Since $t_{i} m a_{k}=t_{i} m$ in $\hat{M}$ if $m \neq 1_{M}$, the edges of $\hat{\Gamma}$ having labels in $A$ are loops at the given vertex except for the edges from $t_{i}$ to $t_{i} a_{k}$. We build $\hat{\Gamma}$ from the $n$ layers by constructing the finite right Cayley digraph $\Gamma_{r}(T, A)$, identifying the vertex $t_{i}$ in this digraph with the vertex $t_{i} 1$ in $\Gamma_{i}$ and adding loops labelled by each element of $A$ at each vertex $t_{i} m, m \neq 1$ of $\Gamma_{i}$. 
Suppose first that $\mathcal{E}_{r}(M)$ is finite and choose a finite subset $\mathfrak{F} \subseteq M$ such that $\Gamma-\mathfrak{F}$ has $\mathcal{E}_{r}(M)$ infinite components. We may assume by Corollary 7 that $1 \in \mathfrak{F}$. For $1 \leq i \leq n$, let $\mathfrak{F}_{i}=\left\{t_{i} m \mid m \in \mathfrak{F}\right\} \subseteq V_{i}$ and let $\hat{\mathfrak{F}}=\bigcup_{i=1}^{n} \mathfrak{F}_{i}$. Observe that $\hat{\mathfrak{F}}$ is finite. Since $\Gamma_{i}-\mathfrak{F}_{i}$ has $\mathcal{E}_{r}(M)$ infinite components for each $i$ and the edges of $\hat{\Gamma}-\hat{\mathfrak{F}}$ having labels in $A$ are all loops, $\hat{\Gamma}-\hat{\mathfrak{F}}$ has $n \mathcal{E}_{r}(M)$ infinite components. This shows that $\hat{\Gamma}$ has at least $n \mathcal{E}_{r}(M)$ ends. Suppose that $\overline{\mathfrak{F}}$ is an arbitrary finite subset of $\hat{M}$. Let

$$
\mathfrak{F}=\mathfrak{F}_{1}=\left\{m \mid t_{i} m \in \overline{\mathfrak{F}} \text { for some } i \text { with } 1 \leq i \leq n\right\}, \quad \mathfrak{F}_{i}=\left\{t_{i} m \mid m \in \mathfrak{F}\right\},
$$

for $1 \leq i \leq n$ and $\hat{\mathfrak{F}}=\bigcup_{i=1}^{n} \mathfrak{F}_{i}$. Observe again that $\hat{\mathfrak{F}}$ is finite. Because $\overline{\mathfrak{F}} \subseteq \hat{\mathfrak{F}}$, we know that $\hat{\Gamma}-\hat{\mathfrak{F}}$ has at least as many infinite components as $\hat{\Gamma}-\overline{\mathfrak{F}}$. It suffices to prove that $\hat{\Gamma}-\hat{\mathfrak{F}}$ has at most $n \mathcal{E}_{r}(M)$ infinite components. If this were not the case, then by the pigeonhole principle, we would have more than $\mathcal{E}_{r}(M)$ infinite components in some $\Gamma_{i}-\mathfrak{F}_{i}$. Since all of these are isomorphic to $\Gamma_{1}-\mathfrak{F}_{1}$, we would then have the contradiction that $\Gamma-\mathfrak{F}$ has more than $\mathcal{E}_{r}(M)$ infinite components.

The argument when $\mathcal{E}_{r}(M)$ is infinite is similar. For every natural number $h$ we can find a finite subset $\mathfrak{F}$ of $M$ such that $1 \in \mathfrak{F}$ and $\Gamma-\mathfrak{F}$ has at least $h$ infinite components. Then, with $\hat{\mathfrak{F}}$, as above, $\hat{\Gamma}-\hat{\mathfrak{F}}$ has at least $h n$ infinite components.

Now write $\Gamma$ for the left Cayley digraph $\ell \Gamma(X, M)$ and $\hat{\Gamma}$ for the left Cayley digraph ${ }_{\ell} \Gamma(A \cup X, \hat{M})$. Write $\Upsilon$ for the left Cayley digraph $\ell_{\ell} \Gamma(A, T)$. For any $m \in M$, define the tower at $m$ to be the the subdiagram $\Upsilon_{m}$ of $\hat{\Gamma}$ having vertex set $V_{m}=\{t m \mid t \in T\}$ and edge set $E_{m}=\left\{f \mid f \in E_{\hat{\Gamma}}, \iota(f)=t m, \tau(f)=a t m, a \in A\right\}$. Then, for every $m \in M$, there is a diagram isomorphism $\phi_{m}: \Upsilon \rightarrow \Upsilon_{m}$ defined on vertices and edges by $\phi_{m}(t)=t m$, and $\phi_{m}(f)$ is the edge from $t m$ to atm if $f$ is the edge from $t$ to at in $\Upsilon$ having label $a$. We can think of constructing $\hat{\Gamma}$ from the set of towers by identifying each vertex $1_{T} m$ in the tower $\Upsilon_{m}$ with the vertex $m$ in $\Gamma$ and for each $x \in X$ and $m \neq 1$ in $M$ adjoining an edge from $t m$ in $\Upsilon_{m}$ to $x m$ in $\Gamma$ having label $x$.

Corresponding to any finite subset $\mathfrak{F}$ of $M$, we define $\hat{\mathfrak{F}}$ to be the finite subset $\hat{\mathfrak{F}}=\left\{t_{i} m \mid 1 \leq i \leq n, m \in \mathfrak{F}\right\}$ of $\hat{M}$. Corresponding to any finite subset $\overline{\mathfrak{F}}$ of $\hat{M}$, let $\mathfrak{F}=\mathfrak{F}_{1}=\left\{m \mid t_{k} m \in \overline{\mathfrak{F}}\right.$ for some $\left.1 \leq k \leq n\right\}$. Since $\overline{\mathfrak{F}} \subseteq \hat{\mathfrak{F}}$, we obtain $\mathrm{e}_{\infty}(\hat{\Gamma})=\mathrm{e}_{\infty}(\Gamma)$ if we show that $\Gamma-\mathfrak{F}$ and $\hat{\Gamma}-\hat{\mathfrak{F}}$ have the same number of infinite components for any finite $\mathfrak{F}$. After discussing some technical details, we construct a bijection $\widehat{-}$ between infinite components $C$ of $\Gamma-\mathfrak{F}$ and infinite components $\hat{C}$ of $\hat{\Gamma}-\hat{\mathfrak{F}}$.

If, for some $x \in X$, the edge $\hat{f} \in E_{\hat{\Gamma}}$ has label $x, \iota(\hat{f})=t m$ and $\tau(\hat{f})=x m$, define the projection of $\hat{f}$ to be the edge $f=\pi(\hat{f})$ in $E_{\Gamma}$ having label $x, \iota(f)=m$ and $\tau(f)=x m$. If the edge $\hat{f} \in E_{\hat{\Gamma}}$ has label $a$ for $a \in A, \iota(\hat{f})=t m$ and $\tau(\hat{f})=a t m$, define the projection, $\pi(\hat{f})$, of $\hat{f}$ to be the empty path in $\Gamma$ at the vertex $m$. We regard the inverse of an empty path at any vertex to be the same empty path. If $\omega=\hat{f}_{1}^{\varepsilon_{1}} \hat{f}_{2}^{\varepsilon_{2}} \cdots \hat{f}_{k}^{\varepsilon_{k}}$, where $\varepsilon_{j}= \pm 1$ and $\hat{f}_{j} \in E_{\hat{\Gamma}}$ for $1 \leq j \leq k$, is a walk in $\hat{\Gamma}-\hat{\mathfrak{F}}$ from $\iota(\omega)$ to $\tau(\omega)$, then by induction on $k,\left(\pi\left(\hat{f}_{1}\right)\right)^{\varepsilon_{1}}\left(\pi\left(\hat{f}_{2}\right)\right)^{\varepsilon_{2}} \cdots\left(\pi\left(\hat{f}_{k}\right)\right)^{\varepsilon_{k}}$ is a walk in $\Gamma-\mathfrak{F}$ from $\iota\left(\left(\pi\left(\hat{f}_{1}\right)\right)^{\varepsilon_{1}}\right)$ to $\tau\left(\left(\pi\left(\hat{f}_{k}\right)\right)^{\varepsilon_{k}}\right)$. Denote this walk by $\pi(\omega)$. In this 
paragraph and the next, walks and paths (allowing negative edges) in a digraph are to be interpreted as walks in the corresponding graph.

If $C$ is an infinite component of $\Gamma-\mathfrak{F}$, define $\hat{C}$ by $\hat{C}=\{t m \mid t \in T, m \in C\}$. Then $\hat{C}$ is an infinite set, since $\{1 m \mid m \in C\} \subseteq \hat{C}$. To see that $\hat{C}$ is connected in $\hat{\Gamma}-\hat{\mathfrak{F}}$, suppose that $t_{i_{1}} m_{1}, t_{i_{2}} m_{2} \in \hat{C}$. Then $m_{1}, m_{2} \in C$, so there is a path from $m_{1}$ to $m_{2}$ in $\Gamma-\mathfrak{F}$ and hence a corresponding path from $1 m_{1}$ to $1 m_{2}$ in $\hat{\Gamma}-\hat{\mathfrak{F}}$. There are also paths, with all edges labelled by elements of $A$ in $\hat{\Gamma}-\hat{\mathfrak{F}}$, from $1 m_{1}$ to $t_{i_{1}} m_{1}$ and from $1 m_{2}$ to $t_{i_{2}} m_{2}$. We compose paths and their inverses to find a path from $t_{i_{1}} m_{1}$ to $t_{i_{2}} m_{2}$. If $C^{*}$ is the component of $\hat{\Gamma}-\hat{\mathfrak{F}}$ which contains $\hat{C}$, let $t^{*} m^{*}$ be any vertex in $C^{*}, \hat{t} \hat{m}$ any vertex in $\hat{C}$ and $\omega$ a path in $\hat{\Gamma}-\hat{\mathfrak{F}}$ from $t^{*} m^{*}$ to $\hat{t} \hat{m}$. Then $\pi(\omega)$ is a walk in $\Gamma-\mathfrak{F}$ from $m^{*}$ to $\hat{m}$, hence $m^{*} \in C, t^{*} m^{*} \in \hat{C}$ and $C^{*}=\hat{C}$. That is, $\hat{C}$ is always a component of $\hat{\Gamma}-\hat{\mathfrak{F}}$. If $C_{1}$ and $C_{2}$ are any two infinite components of $\Gamma-\mathfrak{F}$ and $\omega$ is a path in $\hat{\Gamma}-\hat{\mathfrak{F}}$ from some vertex in $\hat{C}_{1}$ to some vertex in $\hat{C}_{2}$, then $\pi(\omega)$ is a walk in $\Gamma-\mathfrak{F}$ from $\iota(\pi(\omega)) \in C_{1}$ to $\tau(\pi(\omega)) \in C_{2}$, hence $C_{1}=C_{2}$.

To see that ${ }^{\widehat{ }}$ is onto, suppose that $\bar{C}$ is some infinite component of $\hat{\Gamma}-\hat{\mathfrak{F}}$. Define $C$ by $C=\{m \mid t m \in \bar{C}$ for some $t \in T\}$. It is then routine to verify that $C$ is an infinite set, that $C$ is connected, that $C$ is a component of $\Gamma-\mathfrak{F}$ and that $\hat{C}=\bar{C}$.

It is well known that, for any finitely generated group $G$ and finite set $X$ of generators for $G$, the left and right Cayley graphs for $G$ with generating set $X$ are isomorphic, and that such a Cayley graph has $0,1,2$ or else infinitely many ends (see [2, 3, 10-12]). The next six examples illustrate that these conclusions do not hold for left and right Cayley graphs for semigroups and monoids.

EXAmplE 9. For $n \geq 2$, define $A_{n}$ to be the monoid semidirect product $T \rtimes_{\Phi_{0}} M$ where $M$ is the infinite cyclic monoid having generator $x$ and $T$ is the monogenic monoid having monoid presentation $\left\langle t: t^{n}=t^{n-1}\right\rangle$. It is apparent that $M=S^{1}$ where $S$ is the infinite cyclic semigroup having generator $x$. It is easy to see that the left and right Cayley digraphs for $M$ have one end. By the Layer Lemma, $\mathcal{E}_{r}\left(A_{n}\right)=n$ and $\mathcal{E}_{\ell}\left(A_{n}\right)=1$.

To greatly generalize, let $S_{1}$ be any semigroup with $\mathcal{E}_{r}\left(S_{1}\right)=\mathcal{E}_{\ell}\left(S_{1}\right)=1$ and let $S_{2}$ be any semigroup with $n-1$ elements. With $M=S_{1}^{1}$ and $T=S_{2}^{1}$, we have $\mathcal{E}_{r}\left(T \rtimes_{\Phi_{0}} M\right)=n$ and $\mathcal{E}_{\ell}\left(T \rtimes_{\Phi_{0}} M\right)=1$. The monoid $A_{n}$ is arguably the most elementary example of this construction. Another elementary possibility is to replace $T=\left\langle t: t^{n}=t^{n-1}\right\rangle$ by a cyclic group of order $n$ having generator $t$. This has the disadvantage that the element $(t, 1) \in T \rtimes_{\Phi_{0}} M$ is then a left and right unit, preventing us from using this $T \rtimes_{\Phi_{0}} M$ as the monoid $M^{\text {op }}$ in the next example.

EXAMPLE 10. For arbitrary natural numbers $m, n \geq 2$, let $M=A_{n}^{\text {op }}$ and let $T=T_{m}$ now be the the monogenic monoid having monoid presentation $\left\langle t: t^{m}=t^{m-1}\right\rangle$. Then by the Layer Lemma, $\mathcal{E}_{r}\left(T \rtimes_{\Phi_{0}} M\right)=m$ and $\mathcal{E}_{\ell}\left(T \rtimes_{\Phi_{0}} M\right)=n$. For later reference, denote this monoid $T \rtimes_{\Phi_{0}} M$ by $J_{n, m}$. 
EXAMPLE 11. Let $B$ be the monoid having monoid presentation $\left\langle a, b: b a=a^{2}\right\rangle$. We want to show that the right Cayley digraph $\Gamma_{r}(B,\{a, b\})$ has one end while the left Cayley digraph $\ell_{\ell} \Gamma(\{a, b\}, B)$ has infinitely many ends. Every element of $B$ can be uniquely written in the form $a^{j} b^{k}$ where $j, k \geq 0$. For either the right or the left Cayley digraph for $B$, we place the vertex $a^{j} b^{k}$ at the lattice point $(j, k)$ in the first quadrant of the real plane. It is easy to show that $a^{j} b^{k} \cdot a=a^{j+k+1}$ and it is clear that $a^{j} b^{k} \cdot b=a^{j} b^{k+1}$. Similarly, $a \cdot a^{j} b^{k}=a^{j+1} b^{k}$, while $b \cdot b^{k}=b^{k+1}$ when $j=0$, but $b \cdot a^{j} b^{k}=a^{j+1} b^{k}$ when $j>0$.

Suppose first that $\Gamma$ is the right Cayley digraph $\Gamma_{r}(B,\{a, b\})$ and let $\mathfrak{F}$ be a finite set of vertices in $B$. Let $t=\max \left\{j+k \mid a^{j} b^{k} \in \mathfrak{F}\right\}$ and define $\mathfrak{F}_{t}$ by $\mathfrak{F}_{t}=\left\{a^{j} b^{k} \mid\right.$ $j+k \leq t\}$. The graph $\Gamma$ has one end by Corollary 7 if $\Gamma-\mathfrak{F}_{t}$ is connected. Let $a^{j_{1}} b^{k_{1}}, a^{j_{2}} b^{k_{2}}$ be vertices in $\Gamma-\mathfrak{F}_{t}$ and assume without loss of generality that $j_{1}+k_{1} \leq j_{2}+k_{2}$. Then we have a positive or empty path in $\Gamma-\mathfrak{F}_{t}$ from $a^{j_{1}+k_{1}+1}$ to $a^{j_{2}+k_{2}+1}$, with edges labelled by $a$ and edges with label $a$ from $a^{j_{1}} b^{k_{1}}$ to $a^{j_{1}+k_{1}+1}$ and from $a^{j_{2}} b^{k_{2}}$ to $a^{j_{2}+k_{2}+1}$.

Now suppose that $\Gamma$ is the left Cayley digraph $\ell_{\ell} \Gamma(\{a, b\}, B)$. For every natural number $h$, define the subset $\mathfrak{F}_{h}$ of $B$ by $\mathfrak{F}_{h}=\left\{b^{i}: 0 \leq i<h\right\}$. We then observe that $\Gamma-\mathfrak{F}_{h}$ has $h+1$ infinite components $C_{i}$ where $C_{i}$ has vertices $\left\{a^{j} b^{i}: j>0\right\}$ if $0 \leq i<h$ and $C_{h}$ has vertices $\left\{a^{j} b^{k}: j \geq 0, k \geq h\right\}$.

EXAMPLE 12. We have remarked earlier that a Cayley digraph for a semigroup need not be connected. Let $B$ be the monoid from Example 11 and $S$ the subsemigroup of nontrivial elements in $B$. We observe that the right Cayley digraph $\Gamma_{r}(S,\{a, b\})$ is connected, but the left Cayley digraph $\ell_{\ell} \Gamma(\{a, b\}, S)$ has two components.

EXAMPLE 13. Let $B$ be the monoid of Example 11 and observe that the identity element is the only left or right unit in $B$. Let $T=T_{m}$ again be the the monogenic monoid having monoid presentation $\left\langle t: t^{m}=t^{m-1}\right\rangle$. Then by the Layer Lemma, $\mathcal{E}_{r}\left(T \rtimes_{\Phi_{0}} B\right)=m$ and $\mathcal{E}_{\ell}\left(T \rtimes_{\Phi_{0}} B\right)=\infty$.

An element $t$ in a semigroup or a monoid $T$ is a regular element if there is an element $x \in T$ such that $t x t=t$. The semigroup or monoid $T$ is regular if every element of $T$ is regular. An element $t$ in a semigroup or a monoid $T$ is completely regular if there is an element $x \in T$ such that $t x t=t$ and $x t=t x$. A semigroup or monoid $T$ is completely regular if every element of $T$ is completely regular. There are several other equivalent characterizations of completely regular semigroups. For example, a semigroup is completely regular if and only if it is a union of groups. In [9], this is part of Theorem II.1.4, which the authors there describe as the fundamental theorem for the global structure of completely regular semigroups.

EXAMPLE 14 . We know by Corollary 11 that $\mathcal{E}_{r}(I)=\mathcal{E}_{\ell}(I)$ for any finitely generated inverse monoid $I$. In this example and the next, we show that this equality need not hold for completely regular monoids.

Let $G_{1}$ be any finitely generated group having one end. There are many of these, and $G_{1}=\mathbb{Z} \times \mathbb{Z}$ is a standard example. Regard $G_{1}$ as a semigroup with a finite set $X$ 
of semigroup generators and form the monoid $M=\left(G_{1}\right)^{1}$. Then $X$ is a finite set of monoid generators for $M$ and $M$ is both a completely regular monoid and an inverse monoid. For a natural number $n>1$, let $G_{2}$ be any completely regular semigroup with $n-1$ elements. For example, we might take $G_{2}$ to be any group or any semilattice with $n-1$ elements. Let $T=\left(G_{2}\right)^{1}$. Then $T$ is a completely regular monoid with $n$ elements. A routine argument by cases shows that $T \rtimes_{\Phi_{0}} M$ is a (completely) regular monoid whenever $M$ and $T$ are (completely) regular. By the Layer Lemma, we have that $\mathcal{E}_{r}\left(T \rtimes_{\Phi_{0}} M\right)=n$ and $\mathcal{E}_{\ell}\left(T \rtimes_{\Phi_{0}} M\right)=1$. To have one explicit example of this for later reference, let $D_{n}$ be $T \rtimes_{\Phi_{0}} M$ when $G_{1}=\mathbb{Z} \times \mathbb{Z}$ and $G_{2}$ is the cyclic group with $n-1$ elements.

EXAMPLE 15. Let $M=D_{n}^{\text {op }}$ where $D_{n}$ is the completely regular monoid from Example 14. Then $\mathcal{E}_{r}(M)=1$ and $\mathcal{E}_{\ell}(M)=n$. For a natural number $m>1$, let $G_{3}$ be any completely regular semigroup with $m-1$ elements and $T=\left(G_{3}\right)^{1}$. Then $T \rtimes_{\Phi_{0}} M$ is a completely regular monoid and we have $\mathcal{E}_{r}\left(T \rtimes_{\Phi_{0}} M\right)=m$ and $\mathcal{E}_{\ell}\left(T \rtimes_{\Phi_{0}} M\right)=n$ by the Layer Lemma.

We now embark upon a second, more common, construction that will allow us to present some interesting examples of commutative and inverse monoids.

Let $\Lambda$ be an index set and $\left(S_{\lambda}, *_{\lambda}\right)$ be a semigroup for each $\lambda \in \Lambda$. Assume that $S_{\lambda_{1}} \cap S_{\lambda_{2}}=\emptyset$ if $\lambda_{1} \neq \lambda_{2}$ and that 0 is a new element not in $\cup S_{\lambda}$. Define $\vee S_{\lambda}$ to be $\{0\} \cup\left(\bigcup_{\lambda \in \Lambda} S_{\lambda}\right)$ and define a multiplication $*$ on $\vee S_{\lambda}$ by

$$
s * t= \begin{cases}s *_{\lambda} t & \text { if there exists } \lambda \in \Lambda \text { such that } s \in S_{\lambda} \text { and } t \in S_{\lambda}, \\ 0 & \text { otherwise. }\end{cases}
$$

It is easy to see that $*$ is associative. Observe that $\vee S_{\lambda}$ is commutative if and only if every $S_{\lambda}$ is commutative. When $|\Lambda|=2$ and $\left\{S_{\lambda}\right\}=\{A, B\}$, write $A \vee B$ for $\vee S_{\lambda}$ and observe that $A \vee B=B \vee A$.

For a first variant description of $\vee S_{\lambda}$, for any $\lambda$, define $S_{\lambda}^{0}$ to be the semigroup having elements $\{0\} \cup S_{\lambda}$ with the multiplication $*_{\lambda}$ extended by setting $s *_{\lambda} 0=$ $0 *_{\lambda} s=0 *_{\lambda} 0=0$ for all $s \in S_{\lambda}$. Then $\vee S_{\lambda}$ is the 0 -direct union of the semigroups $S_{\lambda}^{0}$. See Clifford and Preston [1, Volume II, p. 13], Howie, [5, p. 71] or Higgins, $[4$, p. 26].

For a second variant description of $\vee S_{\lambda}$, write $S_{0}$ for the one-element semigroup $\{0\}$ and define a multiplication on $\Lambda^{0}=\Lambda \cup\{0\}$ by $0 \cdot 0=\lambda \cdot 0=0 \cdot \lambda=\lambda_{1} \cdot \lambda_{2}=0$ for all $\lambda, \lambda_{1}, \lambda_{2} \in \Lambda$. Then $\Lambda^{0}$ is a rather trivial lower semilattice and $\vee S_{\lambda}$ is a $\Lambda^{0}$ semilattice of the semigroups $S_{\lambda}$. See [1, p. 25, 26], [5, p. 89] or [4, p. 37-39].

LEMMA 12. Suppose that $\Lambda$ is a finite set and that $\left\{S_{\lambda}\right\}_{\lambda \in \Lambda}$ is a set of pairwise disjoint, finitely generated semigroups $S_{\lambda}$. Then $\vee S_{\lambda}$ is finitely generated, $\mathcal{E}_{\ell}\left(\vee S_{\lambda}\right)=$ $\sum_{\lambda \in \Lambda} \mathcal{E}_{\ell}\left(S_{\lambda}\right)$ and $\mathcal{E}_{r}\left(\vee S_{\lambda}\right)=\sum_{\lambda \in \Lambda} \mathcal{E}_{r}\left(S_{\lambda}\right)$.

PROOF. We consider the case for the number of left ends. The case for the number of right ends is dual. If $X_{\lambda}$ is a finite set of generators for $S_{\lambda}$, then $X=\{0\} \cup\left(\cup_{\lambda \in \Lambda} X_{\lambda}\right)$ 
is a finite set of generators for $\vee S_{\lambda}$. Write $\Gamma$ for the left Cayley digraph $\ell \Gamma\left(X, \vee S_{\lambda}\right)$ and write $\Gamma_{\lambda}$ for the left Cayley digraph ${ }_{\ell} \Gamma\left(X_{\lambda}, S_{\lambda}\right)$. If, for each $\lambda \in \Lambda, \mathfrak{F}_{\lambda}$ is a finite subset of $S_{\lambda}$, define $\mathfrak{F}$ to be the finite set $\{0\} \cup\left(\cup \mathfrak{F}_{\lambda}\right)$. The key observation is that if $\lambda_{1} \neq \lambda_{2}, x \in X_{\lambda_{1}}$ and $s \in S_{\lambda_{2}}$, then $x s=0$ in $\vee S_{\lambda}$ and the edge from $x$ to $x s=0$ is not in $\Gamma-\mathfrak{F}$. Hence, the digraph $\Gamma-\mathfrak{F}$ is the disjoint union of the finitely many digraphs $\Gamma_{\lambda}-\mathfrak{F}_{\lambda}$. If $\Gamma_{\lambda}-\mathfrak{F}_{\lambda}$ has $m_{\lambda}$ infinite components then $\Gamma-\mathfrak{F}$ has $\sum m_{\lambda}$ infinite components. If any $\mathcal{E}_{\ell}\left(S_{\lambda}\right)$ is infinite, then we can choose $\mathfrak{F}_{\lambda} \subseteq S_{\lambda}$ for which $m_{\lambda}$ is larger than any given natural number $N$ and hence $\mathcal{E}_{\ell}\left(\vee S_{\lambda}\right)$ must be larger than $N$ also. If $\mathcal{E}_{\ell}\left(S_{\lambda}\right)$ is finite for every $\lambda$, then for every $\lambda$ we can choose $\mathfrak{F}_{\lambda} \subseteq S_{\lambda}$ for which $m_{\lambda}=\mathcal{E}_{\ell}\left(S_{\lambda}\right)$ and conclude that $\mathcal{E}_{\ell}\left(\vee S_{\lambda}\right) \geq \sum_{\lambda \in \Lambda} \mathcal{E}_{\ell}\left(S_{\lambda}\right)$. To prove $\mathcal{E}_{\ell}\left(\vee S_{\lambda}\right) \leq \sum_{\lambda \in \Lambda} \mathcal{E}_{\ell}\left(S_{\lambda}\right)$, it suffices to show that $m \leq \sum_{\lambda \in \Lambda} \mathcal{E}_{\ell}\left(S_{\lambda}\right)$, whenever $\mathfrak{F}$ is a finite subset of $\vee S_{\lambda}$ and $\Gamma-\mathfrak{F}$ has $m$ infinite components. By Corollary 7, we may assume that $0 \in \mathfrak{F}$. For each $\lambda$, define $\mathfrak{F}_{\lambda}$ to be $\mathfrak{F} \cap S_{\lambda}$ and define $m_{\lambda}$ to be the number of infinite components of $\Gamma_{\lambda}-\mathfrak{F}_{\lambda}$. Then, as above, $\Gamma-\mathfrak{F}$ is the disjoint union of the digraphs $\Gamma_{\lambda}-\mathfrak{F}_{\lambda}$ and we obtain $m=\sum m_{\lambda} \leq \sum_{\lambda \in \Lambda} \mathcal{E}_{\ell}\left(S_{\lambda}\right)$.

EXAMPLE 16. For an arbitrary natural number $n$, let $\Lambda$ be an index set with $|\Lambda|=n$, and for each $\lambda \in \Lambda$, let $S_{\lambda}$ be a finitely generated abelian group with $\mathcal{E}_{\ell}\left(S_{\Lambda}\right)=\mathcal{E}_{r}\left(S_{\Lambda}\right)=1$. For example, take $S_{\lambda}$ to be the free abelian group of rank $r_{\lambda} \geq 2$. Let $S=\vee S_{\lambda}$. Then $S$ is a finitely generated, completely regular, commutative inverse semigroup with $\mathcal{E}_{r}(S)=\mathcal{E}_{\ell}(S)=n$.

If $M$ is any monoid, $\operatorname{End}(M)$ is standard notation for the monoid of monoid endomorphisms of $M$. For $m \in M$ and $f \in \operatorname{End}(M)$, mathematicians sometimes find it convenient to write the argument $m$ to the left of the function $f$ and other times find it more convenient to write the argument on the right. Then for $f, g \in \operatorname{End}(M)$, the composition $f g$ has, in general, two different values depending upon which notational convention is followed. We write $\operatorname{End}_{\mathrm{r}}(M)$ for $\operatorname{End}(M)$ when we write functions to the right of their arguments and we write $\operatorname{End}_{\ell}(M)$ for $\operatorname{End}(M)$ when we write functions to the left of their arguments. Then $\operatorname{End}_{\ell}(M)$ and $\operatorname{End}_{\mathrm{r}}(M)$ are duals of each other with respect to the functor ${ }^{\text {op }}$. We use Monic $_{\mathrm{r}}(M)$ for the submonoid of $\operatorname{End}_{\mathrm{r}}(M)$ consisting of one-to-one endomorphisms and $\operatorname{Monic}_{\ell}(M)$ for the submonoid of $\operatorname{End}_{\ell}(M)$ consisting of one-to-one endomorphisms. We note that $\operatorname{Monic}_{\mathrm{r}}(M)$ and $\operatorname{Monic}_{\ell}(M)$ are also dual.

Let $A$ and $B$ be monoids and let $\Phi: A \rightarrow \operatorname{End}_{\ell}(B)$ be a monoid homomorphism. For consistency, we would ordinarily write $\Phi(a)$ for the endomorphism of $B$ which is the image of $a \in A$ and then write $[\Phi(a)](b)$ for the value of this endomorphism at the element $b \in B$. When the monoid homomorphism $\Phi$ is understood from context, we will abbreviate $[\Phi(a)](b)$ as ${ }^{a} b$. Since $\Phi$ is a monoid homomorphism, we have ${ }^{1} b=b$ and ${ }^{a_{1}}\left({ }^{a_{2}} b\right)={ }^{a_{1} a_{2}} b$. Since $\Phi(a)$ is a monoid homomorphism for every $a \in A$, we have ${ }^{a} 1=1$ and ${ }^{a}\left(b_{1} b_{2}\right)=\left({ }^{a} b_{1}\right)\left({ }^{a} b_{2}\right)$. Similarly, if $\Phi: A \rightarrow \operatorname{End}_{\mathrm{r}}(B)$ is a monoid homomorphism, it is often convenient and unambiguous to abbreviate $(b)[(a) \Phi]$ as $b^{a}$ and then observe that $b^{1}=b,\left(b^{a_{1}}\right)^{a_{2}}=b^{a_{1} a_{2}}, 1^{a}=1$, and $\left(b_{1} b_{2}\right)^{a}=\left(b_{1}^{a}\right)\left(b_{2}^{a}\right)$. 
Suppose that $A$ and $B$ are monoids and that $\Phi: A \rightarrow \operatorname{End}_{\mathrm{r}}(B)$ is a monoid homomorphism. We define the monoid semidirect product $A \ltimes_{\Phi} B$ to have elements $\{(a, b) \mid a \in A, b \in B\}$ and multiplication $\left(a_{1}, b_{1}\right)\left(a_{2}, b_{2}\right)=\left(a_{1} a_{2}, b_{1}^{a_{2}} b_{2}\right)$. Similarly, if $\Phi: A \rightarrow \operatorname{End}_{\ell}(B)$ is a monoid homomorphism, we define the monoid semidirect product $B \rtimes_{\Phi} A$ to have elements $\{(b, a) \mid b \in B, a \in A\}$ and multiplication $\left(b_{1}, a_{1}\right)\left(b_{2}, a_{2}\right)=\left(\left(b_{1}\right)\left({ }^{a_{1}} b_{2}\right), a_{1} a_{2}\right)$. It is easily verified that both multiplications are associative with respective identity elements $\left(1_{A}, 1_{B}\right)$ and $\left(1_{B}, 1_{A}\right.$ ). If $\Phi(a)$ (or $\left.(a) \Phi\right)$ is the identity endomorphism $b \mapsto b$ of $B$ for every $a \in A$, then $A \ltimes_{\Phi} B$ is the monoid direct product $A \times B$ while $B \rtimes_{\Phi} A$ is the monoid direct product $B \times A$ and thus $A \ltimes_{\Phi} B \cong B \rtimes_{\Phi} A$ in this case. More generally, we have $A \ltimes_{\Phi} B \cong\left(B^{\text {op }} \rtimes_{\Phi} A^{\text {op }}\right)^{\text {op }}$. If $A$ and $B$ are commutative monoids, then $B^{\mathrm{op}}=B, A^{\mathrm{op}}=A$ and $A \ltimes_{\Phi} B \cong\left(B \rtimes_{\Phi} A\right)^{\mathrm{op}}$.

THEOREM 13. Suppose that $M_{i}$ is a finitely generated infinite monoid for $i=1,2$. If $\Phi: M_{1} \rightarrow \operatorname{Monic}\left(M_{2}\right)$ is a monoid homomorphism, then $\mathcal{E}_{r}\left(M_{1} \ltimes_{\Phi} M_{2}\right)=$ $\mathcal{E}_{\ell}\left(M_{2} \rtimes_{\Phi} M_{1}\right)=1$.

PROOF. Since $M_{2} \rtimes_{\Phi} M_{1} \cong\left(M_{1}^{\mathrm{op}} \ltimes_{\Phi} M_{2}^{\mathrm{op}}\right)^{\mathrm{op}}$, it will be sufficient to prove that $\mathcal{E}_{r}\left(M_{1} \ltimes_{\Phi} M_{2}\right)=1$. Write $M$ for $M_{1} \ltimes_{\Phi} M_{2}$. For $i=1,2$, let $X_{i}$ be a finite set of monoid generators for $M_{i}$. Let $X=\left\{(x, 1) \mid x \in X_{1}\right\} \cup\left\{(1, x) \mid x \in X_{2}\right\}$. Then $X$ is a finite set of monoid generators for $M$.

For the proof below, the reader might find it useful to visualize and then generalize the Cayley digraph for a direct product of two infinite cyclic monoids.

Write $\Gamma$ for the right Cayley digraph $\Gamma_{r}(M, X)$. Since $X_{i}$ is a finite set for $i=1,2$, for every positive integer $n$, there can be only finitely many elements $m_{i} \in M_{i}$ with $L_{X_{i}}\left(m_{i}\right)<n$. Let $\mathfrak{F}$ be a finite subset of $V_{\Gamma}=M$. Since $\mathfrak{F}$ is finite, we may fix a natural number $N=N_{\mathfrak{F}}$, depending upon $\mathfrak{F}$, such that $\mathfrak{F} \subseteq\left\{\left(m_{1}, m_{2}\right) \mid L_{X_{i}}\left(m_{i}\right)<N\right.$ for $i=1,2\}$. As a consequence, an element $\left(m_{1}, m_{2}\right) \in M$ is not in $\mathfrak{F}$ if either $L_{X_{1}}\left(m_{1}\right) \geq N$ or $L_{X_{2}}\left(m_{2}\right) \geq N$.

Suppose that $m \in M_{1}$ and $q, p \in M_{2}$ with $L_{X_{2}}(p)=t>0$. Write $p$ in the form $x_{j_{1}} x_{j_{2}} \cdots x_{j_{t}}$ with $x_{j_{i}} \in X_{2}$ for $1 \leq i \leq t$. Then we have a positive path in $\Gamma$ of length $t$ from $(m, q)$ to $(m, q p)$ with consecutive edges labelled by $\left(1, x_{j_{i}}\right)$ for $1 \leq i \leq t$. For further reference, we may refer to this as the vertical path from $(m, q)$ to $(m, q p)$. If we further assume that $L_{X_{1}}(m) \geq N_{\mathfrak{F}}$, then all of the vertices of this path occur in $\Gamma-\mathfrak{F}$ and hence in the same component of $\Gamma-\mathfrak{F}$.

Suppose that $m \in M_{2}$ and $q, p \in M_{1}$ with $L_{X_{1}}(p)=t>0$. Set $p=x_{j_{1}} x_{j_{2}} \cdots x_{j_{t}}$ with $x_{j_{i}} \in X_{1}$ for $1 \leq i \leq t$. Then there is a positive path in $\Gamma$ of length $t$ from $(q, m)$ to $\left(q p, m^{p}\right)$ with consecutive edges labelled by $\left(x_{j_{i}}, 1\right)$ for $1 \leq i \leq t$. For further reference, we may refer to this as the oblique path from $(q, m)$ to $\left(q p, m^{p}\right)$. We need to discuss hypotheses which will guarantee that this oblique path is in $\Gamma-\mathfrak{F}$.

Let $k$ be a fixed natural number and $x \in X_{1}$ a fixed generator of $M_{1}$. Then $\left\{b \in M_{2} \mid L_{X_{2}}(b)<k\right\}$ is a finite set and hence $\left\{b^{x} \mid b \in M_{2}, L_{X_{2}}(b)<k\right\}$ is also finite. For this $k$ and $x$, define $\bar{k}$ by

$$
\bar{k}=1+\max \left\{L_{X_{2}}\left(b^{x}\right) \mid b \in M_{2}, L_{X_{2}}(b)<k\right\} .
$$


Then if $b \in M_{2}$ and $L_{X_{2}}(b)<k$, we also obtain that $L_{X_{2}}\left(b^{x}\right)<\bar{k}$, or equivalently, if $L_{X_{2}}\left(b^{x}\right) \geq \bar{k}$ then $L_{X_{2}}(b) \geq k$. We may slightly modify our definition for $\bar{k}$ and assume that $\bar{k} \geq k$. Thus, for every natural number $k$, there is a natural number $\bar{k} \geq k$ such that whenever $b \in M_{2}$ and $L_{X_{2}}\left(b^{x}\right) \geq \bar{k}$ then $L_{X_{2}}(b) \geq k$.

Similarly, suppose that $x \in X_{1}$ and that $\ell$ is any natural number. Since $x \Phi$ is oneto-one, there is a natural number $\hat{\ell} \geq \ell$, depending upon $\ell$ and $x$, such that whenever $b \in M_{2}$ and $L_{X_{2}}(b) \geq \hat{\ell}$, then $L_{X_{2}}\left(b^{x}\right) \geq \ell$.

We use the values $\bar{k}$ to keep an oblique path within $\Gamma-\mathfrak{F}$ by our choice of the path's final endpoint. We use the values $\hat{\ell}$ to keep an oblique path within $\Gamma-\mathfrak{F}$ by our choice of the path's initial endpoint.

Consider again oblique paths from $(q, m)$ to $\left(q p, m^{p}\right)$. We regard $q$ as varying over $M_{1}$ and $m$ as varying over $M_{2}$, but we want to fix $p \in M_{1}$ with $L_{X_{1}}(p)=t>0$ and write $p=x_{j_{1}} \cdots x_{j_{t}}$ with $x_{j_{i}} \in X_{1}$ for $1 \leq i \leq t$.

Let $k_{0}=N=N_{\mathfrak{F}}$ and choose $\overline{k_{0}} \geq k_{0}$ such that $L_{X_{2}}(b) \geq k_{0}$ whenever $b \in M_{2}$ with $L_{X_{2}}\left(b^{x_{j_{1}}}\right) \geq \overline{k_{0}}$. Define $k_{1}$ to be $\overline{k_{0}}$. By induction on $i$, for $1 \leq i<t$, choose $\overline{k_{i}} \geq k_{i}$ such that whenever $b \in M_{2}$ with $L_{X_{2}}\left(b^{x_{j_{i+1}}}\right) \geq \overline{k_{i}}$, then $L_{X_{2}}(b) \geq k_{i}$. Define $k_{i+1}$ to be $\bar{k}_{i}$. Then $k_{t}$ depends upon both $N$ and $p$. To emphasize this, we may write $k_{N, p}$ for $k_{t}$. Suppose that for the oblique path from $(q, m)$ to $\left(q p, m^{p}\right)$, we know that $L_{X_{2}}\left(m^{p}\right) \geq k_{t}$. Then it is routine to show, by induction on $i$, for each vertex $\left(q x_{j_{1}} x_{j_{2}} \cdots x_{j_{t-i}}, m^{x_{j_{1}} x_{j_{2}} \cdots x_{j_{t-i}}}\right)$ on the path, that $L_{X_{2}}\left(m^{x_{j_{1}} x_{j_{2}} \cdots x_{j_{t-i}}}\right) \geq k_{t-i} \geq k_{0}=$ $N$ and that $L_{X_{2}}(m) \geq N$, so that all of these vertices are in $\Gamma-\mathfrak{F}$. Thus, the oblique path from $(q, m)$ to $\left(q p, m^{p}\right)$ is in $\Gamma-\mathfrak{F}$ provided that $L_{X_{2}}\left(m^{p}\right) \geq k_{N, p}$.

Let $\ell_{0}=N=N_{\mathfrak{F}}$ and choose $\hat{\ell}_{0} \geq \ell_{0}$ such that $L_{X_{2}}\left(b^{x_{j_{t}}}\right) \geq \ell_{0}$ whenever $b \in M_{2}$ with $L_{X_{2}}(b) \geq \hat{\ell_{0}}$. Define $\ell_{1}$ to be $\hat{\ell_{0}}$. By induction on $i$, for $1 \leq i<t$, choose $\hat{\ell}_{i} \geq \ell_{i}$ such that whenever $b \in M_{2}$ with $L_{X_{2}}(b) \geq \hat{\ell}_{i}$, then $L_{X_{2}}\left(b^{x_{j_{t-i}}}\right) \geq \ell_{i}$. Define $\ell_{i+1}$ to be $\hat{\ell}_{i}$. Then $\ell_{t}$ depends upon both $N$ and $p$. To emphasize this, we may write $\ell_{N, p}$ for $\ell_{t}$. Suppose that for the oblique path from $(q, m)$ to $\left(q p, m^{p}\right)$, we know that $L_{X_{2}}(m) \geq \ell_{t}$. Then it is routine to show, by induction on $i$, that for each vertex $\left(q x_{j_{1}} x_{j_{2}} \cdots x_{j_{i}}, m^{x_{j_{1}} x_{j_{2}} \cdots x_{j_{i}}}\right)$ on the path, that we have $L_{X_{2}}\left(m^{x_{j_{1}} x_{j_{2}} \cdots x_{j_{i}}}\right) \geq \ell_{t-i} \geq$ $\ell_{0}=N$, so that all of these vertices are in $\Gamma-\mathfrak{F}$. Thus, the oblique path from $(q, m)$ to $\left(q p, m^{p}\right)$ is in $\Gamma-\mathfrak{F}$ provided that $L_{X_{2}}(m) \geq \ell_{N, p}$.

Let $C$ be an infinite component of $\Gamma-\mathfrak{F}$. Define sets $I_{C}$ and $J_{C}$ by $I_{C}=\left\{c \in M_{1} \mid(c, d) \in C\right.$ for infinitely many different $\left.d \in M_{2}\right\}$ and $J_{C}=\left\{d \in M_{2} \mid\right.$ $(c, d) \in C$ for infinitely many different $\left.c \in M_{1}\right\}$. Below, we will show that $I_{C}$ and $J_{C}$ are nonempty, that $I_{C}$ is a right ideal in $M_{1}$ and $J_{C}$ is a right ideal in $M_{2}$ and finally that $I_{C}=M_{1}$ and $J_{C}=M_{2}$.

Let $C_{g}$ and $C_{h}$ be two infinite components of $\Gamma-\mathfrak{F}$. To prove that $\Gamma$ has one end, it suffices to show that $C_{g}=C_{h}$ by exhibiting a vertex of $\Gamma$ which is in $C_{g} \cap C_{h}$. Suppose that we know that $J_{C_{g}}=M_{2}$. Then $1 \in J_{C_{g}}$, so there are infinitely many $c \in M_{1}$ with $(c, 1) \in C_{g}$. We may then choose $p \in M_{1}$ with $(p, 1) \in C_{g}$ and $L_{X_{1}}(p) \geq N_{\mathfrak{F}}$. Suppose also that we know that $I_{C_{h}}=M_{1}$. Then $1 \in I_{C_{h}}$, so there are infinitely many $d \in M_{2}$ with $(1, d) \in C_{h}$. Choose $m \in M_{2}$ with $(1, m) \in C_{h}$ and $L_{X_{2}}(m) \geq \ell_{N, p}$. Since $L_{X_{1}}(p) \geq N_{\mathfrak{F}}$, the vertical path, labelled by $m^{p}$, in $\Gamma$ from 
$(p, 1)$ to $\left(p, m^{p}\right)$ is in $\Gamma-\mathfrak{F}$ and hence in $C_{g}$. Since $L_{X_{2}}(m) \geq \ell_{N, p}$, the oblique path in $\Gamma$ from $(1, m)$ to $\left(p, m^{p}\right)$ is in $\Gamma-\mathfrak{F}$ and hence in $C_{h}$. Then the vertex $\left(p, m^{p}\right)$ is in both $C_{g}$ and $C_{h}$, so $C_{g}=C_{h}$.

We want to show that, for any infinite component $C$ of $\Gamma-\mathfrak{F}$, the set $I_{C}$ is nonempty, is a right ideal in $M_{1}$, and is all of $M_{1}$.

If it were the case that there were only finitely many elements $c \in M_{1}$ with $(c, d) \in$ $C$ for some $d \in M_{2}$, then, since $C$ is infinite, for at least one such element, $\hat{c}$, there must be infinitely many different $d \in M_{2}$ with $(\hat{c}, d) \in C$ and then $\hat{c} \in I_{C}$. Suppose then that there are infinitely many different elements $c \in M_{1}$ with $(c, d) \in C$ for some $d \in M_{2}$. Then there is an element $(\hat{c}, \hat{\delta}) \in C$ with $L_{X_{1}}(\hat{c}) \geq N_{\mathfrak{F}}$. The vertical path from $(\hat{c}, 1)$ to $(\hat{c}, \hat{\delta})$ is in $C$, so $(\hat{c}, 1) \in C$. But then the vertical path from $(\hat{c}, 1)$ to $(\hat{c}, d)$ is in $C$ for every $d \in M_{2}$, so $\hat{c} \in I_{C}$.

To show that $I_{C}$ is a right ideal in $M_{1}$, it suffices to show that $c x \in I_{C}$ whenever $c \in I_{C}$ and $x \in X_{1}$. Choose $\hat{\ell}=\ell_{N, x}$ such that $L_{X_{2}}\left(d^{x}\right) \geq N$ whenever $d \in M_{2}$ and $L_{X_{2}}(d) \geq \hat{\ell}$. Since $c \in I_{C}$, there are infinitely many $d \in M_{2}$ with $(c, d) \in C$ and hence, for infinitely many of these, $L_{X_{2}}(d) \geq \hat{\ell}$. For each of these, the edge with label $(x, 1)$ from $(c, d)$ to $\left(c x, d^{x}\right)$ is in $C$. Since $x \Phi$ is one-to-one, these values for $d^{x}$ are distinct and $c x \in I_{C}$.

Since $I_{C}$ is a right ideal in $M_{1}$, we will obtain $I_{C}=M_{1}$ if we show that $1=1_{M_{1}} \in$ $I_{C}$. Suppose that $\hat{c} \in I_{C}$ with $L_{X_{1}}(\hat{c}) \geq N_{\mathfrak{F}}$. Then, as above, $(\hat{c}, 1) \in C$ and $(\hat{c}, d) \in C$ for every $d \in M_{2}$. There are infinitely many $d \in M_{2}$ with $L_{X_{2}}(d) \geq \ell_{N, \hat{c}}$. For each of these, $\left(\hat{c}, d^{\hat{c}}\right) \in C$ and there is the oblique path from $(1, d)$ to $\left(\hat{c}, d^{\hat{c}}\right)$ in $C$. If $I_{C}$ is infinite, there is always some $\hat{c} \in I_{C}$ with $L_{X_{1}}(\hat{c}) \geq N_{\mathfrak{F}}$. Suppose instead that $I_{C}$ is finite. Then, since $I_{C}$ is a subsemigroup of $M_{1}$, it must contain an idempotent $e$. Then there are infinitely many $d \in M_{2}$ with $(e, d) \in C$, hence infinitely many such $d$ with both $L_{X_{2}}(d) \geq \ell_{N, e}$ and $L_{X_{2}}\left(d^{e}\right) \geq k_{N, e}$. For the latter, we use the hypothesis that $e \Phi$ is one-to-one. Since $L_{X_{2}}(d) \geq \ell_{N, e}$, for each such $d$, the oblique path from $(e, d)$ to $\left(e \cdot e, d^{e}\right)=\left(e, d^{e}\right)$ is in $C$ for each of these infinitely many $d$. Since $L_{X_{2}}\left(d^{e}\right) \geq k_{N, e}$, the oblique path from $(1, d)$ to $\left(e, d^{e}\right)$ is also in $C$ for each of these infinitely many $d$ and we see that $1_{M_{1}} \in I_{C}$.

We want to show, for any infinite component $C$ of $\Gamma-\mathfrak{F}$, that $1_{M_{2}} \in J_{C}$, and that $J_{C}$ is a right ideal in $M_{2}$ and hence is all of $M_{2}$. We now know that $I_{C}=M_{1}$, so there are infinitely many elements $c \in I_{C}$ with $L_{X_{1}}(c) \geq N_{\mathfrak{F}}$ and for each such $c$ an element $d_{c} \in M_{2}$ with $\left(c, d_{c}\right) \in C$. For each such $c$, the vertical path from $(c, 1)$ to $\left(c, d_{c}\right)$ is in $C$, so we have infinitely many different $c$ with $(c, 1) \in C$ and $1 \in J_{C}$. Suppose that $d \in J_{C}$ and that $x \in X_{2}$. Then, for infinitely many $c \in M_{1},(c, d) \in C$, and there are thus infinitely many such $c$ with $L_{X_{1}}(c) \geq N_{\mathfrak{F}}$. For those $c$ with $L_{X_{1}}(c) \geq N_{\mathfrak{F}}$, we can be sure that the edge labelled $(1, x)$ from $(c, d)$ to $(c, d x)$ is in $C$, so $(c, d x) \in C$ for infinitely many $c$ and $d x \in J_{C}$.

EXAMPLE 17. In this example, we want to show that, in the previous theorem, the hypothesis that $\Phi$ has its range in Monic $\left(M_{2}\right)$ rather than just in $\operatorname{End}\left(M_{2}\right)$ is necessary. We also see that the second conclusion of the Layer Lemma need not hold if $T$ 


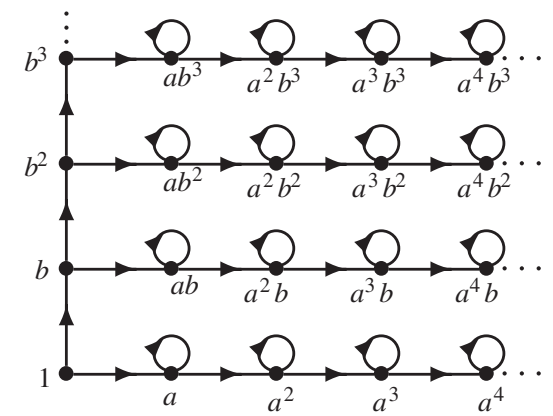

Left digraph

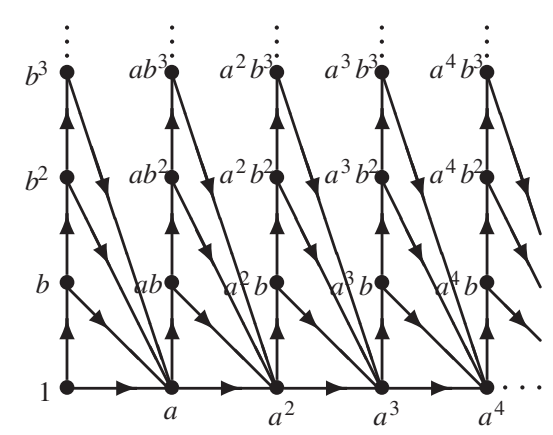

Right digraph

FIGURE 3. Left and right Cayley digraphs for $A \ltimes_{\Phi_{0}} B$.

is an infinite monoid. Let $A=\langle a\rangle$ and $B=\langle b\rangle$ be free monogenic monoids and $M=A \ltimes \Phi_{0} B$. Here $a \Phi_{0}=\theta_{B}$ where $b^{m} \theta_{B}=1_{B}$ for every nonnegative integer $m$, hence $\theta_{B}$ is not one-to-one.

Then $M$ has monoid presentation $\langle a, b: b a=a\rangle$ and every element of $M$ can be represented by a unique word in the form $a^{n} b^{m}$ for nonnegative integers $m, n$. We illustrate the right and left Cayley digraphs for $A \ltimes \Phi_{0} B$ in Figure 3. We remark that

$$
\mathcal{E}_{r}\left(A \ltimes_{\Phi_{0}} B\right)=\mathcal{E}_{\ell}\left(A \ltimes_{\Phi_{0}} B\right)=\mathcal{E}_{r}\left(B \rtimes_{\Phi_{0}} A\right)=\mathcal{E}_{\ell}\left(B \rtimes_{\Phi_{0}} A\right)=\infty .
$$

Since $M^{\text {op }} \approx B \rtimes_{\Phi_{0}} A$, we see that $\mathcal{E}_{\ell}\left(B \rtimes_{\Phi_{0}} A\right)=\mathcal{E}_{r}(M)=\infty$. This shows that it is not necessarily the case that $\mathcal{E}_{\ell}\left(B \rtimes_{\Phi_{0}} A\right)=1$, as in Theorem 13, if $\Phi=\Phi_{0}$ nor that $\mathcal{E}_{\ell}\left(B \rtimes_{\Phi_{0}} A\right)=\mathcal{E}_{\ell}(A)$, as in the Layer Lemma, if $B$ is infinite.

EXAMPLE 18. Our initial impression was that, with the hypotheses of the previous theorem, we should also be able to prove that $\mathcal{E}_{\ell}\left(M_{1} \ltimes_{\Phi} M_{2}\right)=\mathcal{E}_{r}\left(M_{2} \rtimes_{\Phi} M_{1}\right)=1$. This is not valid. Here, we give an example where $\Phi: M_{1} \rightarrow \operatorname{Monic}\left(M_{2}\right)$ is a monoid homomorphism but $\mathcal{E}_{\ell}\left(M_{1} \ltimes_{\Phi} M_{2}\right)=\mathcal{E}_{r}\left(M_{2} \rtimes_{\Phi} M_{1}\right)=\infty$.

Let $M_{1}=\langle a\rangle$ be the free cyclic monoid with generator $a$. Let $M_{2}$ be the monoid having monoid presentation $\left\langle b, t \mid b t=t, t^{2}=t\right\rangle$. Then one can show that every element of $M_{2}$ has a unique representative of the form $t^{\varepsilon} b^{n}$ where $\varepsilon$ has value 0 or 1 and $n$ is a nonnegative integer. We want to define a semidirect product, $M_{1} \ltimes_{\Phi} M_{2}$, so we write functions to the right of their arguments. Define $\alpha: M_{2} \rightarrow M_{2}$ by $b^{n} \alpha=b^{n}$ and $\left(t b^{n}\right) \alpha=t b^{n+1}$. Then $\alpha$ is a monoid homomorphism and is one-to-one. Since $M_{1}$ is free on $a$, we obtain a monoid homomorphism $\Phi: M_{1} \rightarrow \operatorname{Monic}_{\mathrm{r}}\left(M_{2}\right)$ by setting $a \Phi=\alpha$. Then the semidirect product $M=M_{1} \ltimes_{\Phi} M_{2}$ has elements $\left\{\left(a^{m}, t^{\varepsilon} b^{n}\right) \mid\right.$ $m \geq 0, n \geq 0, \varepsilon=0,1\}$ and is generated by $(a, 1),(1, b)$ and $(1, t)$. If we identify $(a, 1),(1, b)$ and $(1, t)$ with $a, b$ and $t$, respectively, then $M$ has a monoid presentation $\left\langle a, b, t \mid t a=a t b, b a=a b, b t=t, t^{2}=t\right\rangle$ and every element of $M$ has a unique representative of the form $a^{m} t^{\varepsilon} b^{n}$ with $m \geq 0, n \geq 0, \varepsilon=0,1$. Write just $\Gamma$ for the 
left Cayley digraph ${ }_{\ell} \Gamma(\{a, b, t\}, M)$. We want to show that $\mathrm{e}_{\infty}(\Gamma)=\infty$. It is easily verified that

$$
\begin{gathered}
a \cdot\left(a^{m} t^{\varepsilon} b^{n}\right)=a^{m+1} t^{\varepsilon} b^{n}, \quad b \cdot\left(a^{m} b^{n}\right)=a^{m} b^{n+1}, \\
b \cdot\left(a^{m} t b^{n}\right)=a^{m} t b^{n}, \quad t \cdot\left(a^{m} b^{n}\right)=a^{m} t b^{m+n}, \quad t \cdot\left(a^{m} t b^{n}\right)=a^{m} t b^{n} .
\end{gathered}
$$

We thus account for all of the edges in $\Gamma$. To show that $\mathrm{e}_{\infty}(\Gamma)=\infty$, it suffices to find, for every natural number $k$, a finite subset $\mathfrak{F}_{k} \subseteq V_{\Gamma}$ such that $\left|\mathfrak{C}_{\infty}\left(\Gamma-\mathfrak{F}_{k}\right)\right|>k$.

Let $\mathfrak{F}_{k}=\left\{a^{m} t b^{n} \mid 0 \leq m \leq k, 0 \leq n \leq k\right\}$. For $0 \leq i \leq k$, let $C_{i}$ be the full subgraph of $\Gamma$ on the set $\left\{a^{m} t b^{i} \mid m>k\right\}$. We will be done when we show that each $C_{i}$ is a component of $\Gamma-\mathfrak{F}_{k}$. (There is one more component of $\Gamma-\mathfrak{F}_{k}$, but we need not concern ourselves with it.) $C_{i}$ is connected since we have an edge labelled by $a$ from $a^{m} t b^{i}$ to $a^{m+1} t b^{i}$ for each $m>k$. For a vertex $v=a^{m} t b^{i}$ in $C_{i}$, the other edges having $v$ as an initial vertex are loops labelled by $b$ and $t$. Examining the account of the edges in $\Gamma$ in the previous paragraph, we see that the only other edges in $\Gamma$ having a vertex $v=a^{m} t b^{i}$ as a terminal vertex are edges with label $t$ from $a^{m} b^{n}$ to $a^{m} t b^{m+n}$. But such an edge cannot have its terminal vertex in $C_{i}$ if $m+n=i \leq k$ and $m>k$.

Corollary 14. Suppose that $G_{i}$ is a finitely generated infinite group for $i=1,2$. If $\Phi: G_{1} \rightarrow \operatorname{Aut}\left(G_{2}\right)$ is a group automorphism, then the group semidirect product $G_{2} \rtimes_{\Phi} G_{1}$ has one end.

PROOF. Group automorphisms are endomorphisms and are one-to-one.

COROllary 15. Suppose, for $i=1,2$, that $M_{i}$ is an infinite monoid with a finite set of monoid generators $X_{i}$. Let $M=M_{1} \times M_{2}$ be the monoid direct product. Then $\mathcal{E}_{r}(M)=\mathcal{E}_{\ell}(M)=1$.

PROOF. The direct product is a special case of Theorem 13 where $\Phi$ takes each element of $M_{1}$ to the identity automorphism of $M_{2}$.

EXAMPLE 19. Let $m, n, \hat{m}, \hat{n}$ be arbitrary natural numbers. We construct a monoid $M$ having a submonoid $J$ such that $\mathcal{E}_{r}(M)=\hat{m}, \mathcal{E}_{\ell}(M)=\hat{n}, \mathcal{E}_{r}(J)=m$ and $\mathcal{E}_{\ell}(J)=n$. Let $J=J_{n, m}$ be the monoid of Example 10 and let $P$ be the direct product of $J$ with any infinite monoid of the form $S^{1}$ for some semigroup $S$. Then $P$ contains a submonoid which is isomorphic to $J$, and $P$, like $J$ and $S^{1}$, contains no nontrivial left or right units. By the corollary, $\mathcal{E}_{r}(P)=\mathcal{E}_{\ell}(P)=1$. For any natural number $k$ and generator $y$, let $T(y, k)$ be the the monogenic monoid having monoid presentation $\left\langle y: y^{k}=y^{k-1}\right\rangle$. The monoid $M=T(u, \hat{m}) \rtimes_{\Phi_{0}}\left(T(v, \hat{n}) \rtimes_{\Phi_{0}} P^{\text {op }}\right)^{\text {op }}$ contains a submonoid isomorphic to $P$. With two applications of the Layer Lemma, we obtain $\mathcal{E}_{r}(M)=\hat{m}$ and $\mathcal{E}_{\ell}(M)=\hat{n}$.

\section{Subsemigroups of free semigroups}

Our principal results in this section are about the number of ends of subsemigroups of free semigroups. Theorem 18 says that every commutative subsemigroup of 
a free semigroup has one end. Theorem 20 says that every finitely generated noncommutative subsemigroup of a free semigroup has infinitely many ends. The analogous results for submonoids of free monoids follow immediately by adjoining the empty word. The next two lemmas will be used in the proof. We regard the first lemma as elementary and well known. The group versions in [6, Exercise 1.4.6] and [7, Proposition I.2.17] are easily modified to obtain the semigroup version.

Lemma 16. Suppose that $F$ is the free semigroup on the alphabet $A$ and that $u$, $v \in F$. If $u v=v u$, then there are natural numbers $m, n$ and an element $w \in F$ such that $u=w^{m}$ and $v=w^{n}$.

LEMMA 17. If $S$ is any subsemigroup of the additive semigroup $\mathbb{N}$ of natural numbers, then $\mathcal{E}_{\ell}(S)=\mathcal{E}_{r}(S)=1$.

PROOF. Let $S$ be a subsemigroup of the additive semigroup $\mathbb{N}$. Since $S$ is commutative, it is clear from Proposition 10 that $\mathcal{E}_{\ell}(S)=\mathcal{E}_{r}(S)$ when these are defined. Since we have only defined $\mathcal{E}_{\ell}(S)$ and $\mathcal{E}_{r}(S)$ for finitely generated semigroups $S$, we need to show that $S$ is finitely generated.

If all of the elements of $S$ are divisible by some natural number $d>1$, assume that $d$ is the largest natural number dividing all of the elements of $S$ and define $\hat{S}$ by $\hat{S}=\{s / d \mid s \in S\}$. Then $\hat{S}$ is a subsemigroup of $\mathbb{N}$ which is isomorphic to $S$. We can replace $S$ by $\hat{S}$ and assume that the greatest common divisor of the set of all elements of $S$ is 1 . It is easy to see, using elementary number theory, that $S$ then contains all but finitely many natural numbers. If we write $n_{0}-1$ for the greatest natural number that is not in $S$, then we may write $S=X_{0} \cup\left\{n \in \mathbb{N} \mid n \geq n_{0}\right\}$ for some finite set $X_{0} \subseteq \mathbb{N}$. We then see that $S$ is generated by the finite set $X=X_{0} \cup\left\{n \in \mathbb{N} \mid n_{0} \leq n<2 n_{0}\right\}$. Write $\Gamma$ for $\Gamma_{r}(S, X)$.

Now let $\mathfrak{F}$ be any finite subset of vertices of $\Gamma$, let $m$ be the largest element in $\mathfrak{F}$ and choose any $k \in \mathbb{N}$ which satisfies $m<k n_{0}$. Then the set $C=\left\{n \mid n \geq(k+1) n_{0}\right\}$ is an infinite subset of $\Gamma-\mathfrak{F}$ having a finite complement in $\mathbb{N}$, so we will be done when we show that $C$ is contained in the component of $\Gamma-\mathfrak{F}$ which contains $k n_{0}$. For an arbitrary element $n \in C$, write $n=q n_{0}+r$, where $0 \leq r<n_{0}$ and $q \geq k+1$. Then we have a path of length $q-k$ from $k n_{0}$ to $n$ in $\Gamma-\mathfrak{F}$ having 1 edge labelled by $n_{0}+r$ and $q-k-1$ edges labelled by $n_{0}$.

THEOREM 18. If $S$ is a commutative subsemigroup of a free semigroup, then $\mathcal{E}_{\ell}(S)=\mathcal{E}_{r}(S)=1$.

PROOF. Using Lemma 16, it can be shown that any set of pairwise commuting elements in a free semigroup must consist of powers of a single word. Hence $S$ is isomorphic to a subsemigroup of $\mathbb{N}$ and the conclusion follows from Lemma 17.

LEMMA 19. Let $F$ be the free semigroup on the alphabet $A$ and let $S$ be a finitely generated subsemigroup of $F$ with finite set of generators $X$. Let $\Gamma$ be the right Cayley graph $\Gamma_{r}(S, X)$. If $\mathfrak{F}$ is a finite subset of $S$ and $w$ is a element of $S-\mathfrak{F}$, write $C_{w}$ for 
the component of $\Gamma-\mathfrak{F}$ containing $w$. If the length, $L_{A}(w)$, of $w$ on the alphabet $A$ is minimal among elements of $S-\mathfrak{F}$, then $w$ is a prefix of every vertex in $C_{w}$.

PROOF. Let $u$ be an arbitrary element of $C_{w}$. We use induction on the length of a path in $C_{w}$ from $w$ to $u$ to show that $w$ is a prefix of $u$. The base case, where $u=w$, is obvious. Suppose that $u \neq w$ and write $p$ for the vertex before $u$ on a path from $w$ to $u$ in $C_{w}$. By the induction hypothesis, $w$ is a prefix of $p$ and $p=w p^{\prime}$ for some, possibly empty, word $p^{\prime}$. If $u=p x=w p^{\prime} x$ for some generator $x \in X$, we are done. If $w p^{\prime}=p=u x$, then by the minimality of $L_{A}(w)$ among elements of $S-\mathfrak{F}$, it must be true that $L_{A}(w) \leq L_{A}(u)$ and again $w$ is a prefix of $u$.

THEOREM 20. If $S$ is a finitely generated subsemigroup of a free semigroup and $S$ is not commutative, then $\mathcal{E}_{\ell}(S)=\mathcal{E}_{r}(S)=\infty$.

Proof. As in Lemma 19, we write $F$ for the free semigroup on the alphabet $A$, write $X$ for some finite set of generators for the subsemigroup $S$ of $F$, and $\Gamma$ for the right Cayley graph $\Gamma_{r}(S, X)$. We have hypothesized that $S$ is not commutative and we choose two elements $x, y \in S$ such that $x y \neq y x$. It suffices to exhibit, for every natural number $n$, a finite subset $\mathfrak{F}$ of $S$ such that $\Gamma-\mathfrak{F}$ has at least $n+1$ infinite components. Let $n \in \mathbb{N}$ be arbitrary. For integers $0 \leq i \leq n$, define the element $w_{i} \in S$ by $w_{i}=x^{i} y x^{n-i}$. All of the $w_{i}$ have the same length, $n L_{A}(x)+L_{A}(y)$. For notational convenience, we write $\ell$ for this length. By the cancellative properties in the free semigroup $S$, all of the $w_{i}$ are distinct. Let $\mathfrak{F}=\left\{s \in S \mid L_{A}(s)<\ell\right\}$. By construction, each $w_{i}$ must have minimal length in $S-\mathfrak{F}$ and, using Lemma 19, we see that elements $w_{i}$ must occur in distinct components of $\Gamma-\mathfrak{F}$. It is easily seen that these components are infinite.

\section{Acknowledgement}

We thank the referee very much for comments on the treatment in Section 4. The proofs are now much briefer and cleaner as a result.

\section{References}

[1] A. H. Clifford and G. B. Preston, The Algebraic Theory of Semigroups, Mathematical Surveys, 7 (American Mathematical Society, Providence, RI, 1961).

[2] D. E. Cohen, Groups of Cohomological Dimension One, Lecture Notes in Mathematics, 245 (Springer, Berlin, 1972).

[3] M. J. Dunwoody, 'The ends of finitely generated groups', J. Algebra 12 (1969), 339-344.

[4] P. M. Higgins, Techniques of Semigroup Theory (Oxford University Press, Oxford, 1992).

[5] J. M. Howie, An Introduction to Semigroup Theory (Academic Press, London, 1976).

[6] R. C. Lyndon and P. E. Schupp, Combinatorial Group Theory, Ergebnisse der Mathematik und ihrer Grenzgebiete, 89 (Springer, Berlin, 1977).

[7] W. Magnus, A. Karrass and D. Solitar, Combinatorial Group Theory, Second Revised Edition (Dover Publications, New York, 1976).

[8] M. Petrich, Inverse Semigroups (Wiley, New York, 1984).

[9] M. Petrich and N. R. Reilly, Completely Regular Semigroups (Wiley, New York, 1999). 
[10] P. E. Schupp, 'Groups and graphs', Math. Intelligencer 1 (1979), 205-214.

[11] J. Stallings, 'On torsion-free groups with infinitely many ends', Ann. of Math. 88 (1968), 312-334.

[12] J. Stallings, Group Theory and Three-dimensional Manifolds, Yale Monographs, 4 (Yale University Press, New Haven, CT, 1971).

DAVID A. JACKSON, Department of Mathematics, Saint Louis University, St. Louis, MO 63103, USA

e-mail: jacksoda@slu.edu

VESNA KILIBARDA, Department of Mathematics, Indiana University Northwest, Gary, IN 46408, USA

e-mail: vkilibar@iun.edu 